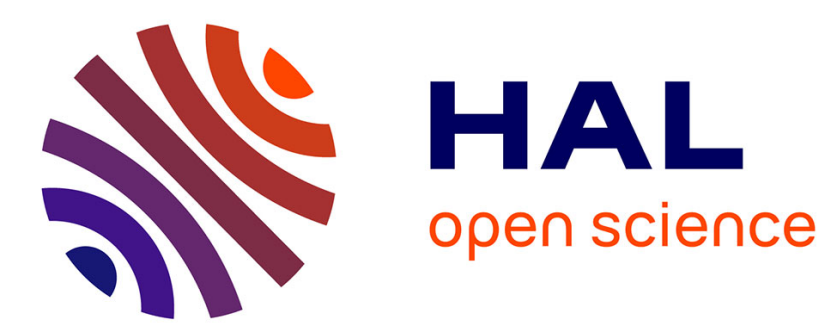

\title{
Periodic Solutions of the 1D Vlasov-Maxwell System with Boundary Conditions
}

\author{
Mihai Bostan
}

\section{To cite this version:}

Mihai Bostan. Periodic Solutions of the 1D Vlasov-Maxwell System with Boundary Conditions. RR3554, INRIA. 1998. inria-00073129

\section{HAL Id: inria-00073129 \\ https://hal.inria.fr/inria-00073129}

Submitted on 24 May 2006

HAL is a multi-disciplinary open access archive for the deposit and dissemination of scientific research documents, whether they are published or not. The documents may come from teaching and research institutions in France or abroad, or from public or private research centers.
L'archive ouverte pluridisciplinaire HAL, est destinée au dépôt et à la diffusion de documents scientifiques de niveau recherche, publiés ou non, émanant des établissements d'enseignement et de recherche français ou étrangers, des laboratoires publics ou privés. 


\title{
Periodic solutions of the 1D Vlasov-Maxwell system with boundary conditions
}

\author{
Mihai Bostan
}

\section{$\mathbf{N}^{\circ} 3554$}

November 1998

THÈME 4 



\title{
Periodic solutions of the 1D Vlasov-Maxwell system with boundary conditions
}

\author{
Mihai Bostan \\ Thème 4 - Simulation et optimisation \\ de systèmes complexes \\ Projet CAIMAN \\ Rapport de recherche $\mathrm{n}^{\circ} 3554$ - November 1998 - 39 pages
}

\begin{abstract}
We study the 1D Vlasov-Maxwell system with time periodic boundary conditions in its classical and relativistic form. For small data we prove existence of weak periodic solutions. It is necessary to impose non vanishing conditions for the incoming velocities in order to control the life-time of particles in the domain. In order to preserve the periodicity, another condition of vanishing the time average of the incoming current is imposed.
\end{abstract}

Key-words: Maxwell equations - Vlasov equation - Schauder's fixed point theorem. 


\section{Solutions périodiques du système de Vlasov-Maxwell 1D avec conditions aux limites}

Résumé : Nous présentons dans ce rapport l'existence d'une solution périodique pour le système de Vlasov-Maxwell dans une dimension d'espace, avec des conditions limites périodiques en temps. Les cas classique et relativist sont étudiés. Afin de préserver la périodicité des solutions, une condition d'annulation de la moyenne en temps du courant est imposée.

Mots-clés : Équations de Maxwell - Équation de Vlasov - Théorème du point fixe de Schauder. 


\section{Contents}

$\begin{array}{ll}\text { Introduction } & 4\end{array}$

1 Definitions and bounds for the Vlasov equation $\quad 7$

1.1 Weak and mild solution of the Vlasov equation . . . . . . . . 7

1.2 Estimation of the life-time of particles . . . . . . . . . . 11

2 Weak periodic solutions for the modified 1D Vlasov-Maxwell $\begin{array}{ll}\text { system. } & \mathbf{1 7}\end{array}$

2.1 Fixed point for the regularized problem . . . . . . . . . 18

3 Weak periodic solutions for the classical 1D Vlasov-Maxwell system. $\quad 28$

4 Weak periodic solutions for the relativistic 1D Vlasov-Maxwell system. 


\section{Introduction}

The coupled nonlinear system presented by the Vlasov-Maxwell equations is a classical model in the kinetic theory of plasma. The main assumption underlying the model is that collisions are so rare that they may be neglected. In one dimension of space the Vlasov-Maxwell system $(V M)$ writes:

$$
\begin{gathered}
\partial_{t} f+v_{x} \cdot \partial_{x} f+\frac{q}{m}\left(E_{x}+v_{y} \cdot B_{z}\right) \cdot \partial_{v_{x}} f+\frac{q}{m}\left(E_{y}-v_{x} \cdot B_{z}\right) \cdot \partial_{v_{y}} f=0, \\
\left(t, x, v_{x}, v_{y}\right) \in \mathbb{R _ { t } \times ] 0 , L [ \times \mathbb { R } _ { v } ^ { 2 } ,} \\
\left.\partial_{t} E_{x}=-\frac{1}{\varepsilon} j_{x}:=-\frac{1}{\varepsilon} \int_{v} v_{x} f\left(t, x, v_{x}, v_{y}\right) d v, \quad(t, x) \in \mathbb{R}_{t} \times\right] 0, L[, \\
\left.\partial_{t} E_{y}+c^{2} \partial_{x} B_{z}=-\frac{1}{\varepsilon} j_{y}:=-\frac{1}{\varepsilon} \int_{v} v_{y} f\left(t, x, v_{x}, v_{y}\right) d v, \quad(t, x) \in \mathbb{R}_{t} \times\right] 0, L[, \\
\partial_{t} B_{z}+\partial_{x} E_{y}=0, \\
\left.(t, x) \in \mathbb{R}_{t} \times\right] 0, L[.
\end{gathered}
$$

The variables $\left(t, x, v_{x}, v_{y}\right)$ are respectively the time, the position and the velocity. The non-negative function $f$ is the distribution of the charged particles of charge $q$ and mass $m,\left(E_{x}, E_{y}, B_{z}\right)$ is the electro-magnetic field, $\varepsilon$ is the electric permitivity of the vacuum and $c$ is the light velocity in the vacuum. A reduced description of the plasma is obtained by neglecting the magnetic field $B$. The associated model constitutes the Vlasov-Poisson system $(V P)$ and it can be justified ( at least for small time ) by a non-relativistic limit [18]. The main result in this field has been obtained in 1989 by R.J.DiPerna and P.L.Lions [14]. They prove existence of global weak solutions for the Cauchy problem with arbitrary data. The global existence of strong solution is still an open problem. In the case of the Vlasov-Poisson system weak global solution for the Cauchy problem has been obtained by Arsenev [16]. Existence of strong solution in 2D is a result due to Degond [19] and Ukai Ohabe [20]. The same result in 3D has been proved by Pfaffelmoser [8]. For applications like vacuum diodes, tube discharges, cold plasma, solar wind, satellite ionisation, thrusters, 
etc... boundary conditions have to be taken into account. For the transient regime global weak solutions of the Vlasov-Maxwell system has been proved to exist by Y.Guo [6] and independently by M.Bezart [15]. The same problem for the Vlasov-Poisson system has been investigated by Y.Guo [5] and N.Ben Abdallah [10]. Permanent regimes are particularly important. They are of two types and they are modeled by stationary solutions or time periodic solutions for boundary value problems. Results concerning stationary problems can be found in the paper of C.Greengard P.A.Raviart [1] for the Vlasov-Poisson system in $1 \mathrm{D}$ and in the paper of F.Poupaud for the Vlasov-Maxwell system [4]. For the periodic problems, results can be found in [12]. We now describe precisely the boundary condition. Let ] $0, L$ [ representing the device geometry. We denote by $\Sigma^{-}$the set of initial positions in phase space of incoming particles :

$$
\begin{aligned}
\Sigma^{-}= & \left\{\left(0, v_{x}, v_{y}\right) \mid v_{x}>0, v_{y} \in \mathbb{R}\right\} \\
\cup & \left\{\left(L, v_{x}, v_{y}\right) \mid v_{x}<0, v_{y} \in \mathbb{R}\right\} .
\end{aligned}
$$

The distribution of incoming particles is prescribed :

$$
f= \begin{cases}g_{0}, & \left(t, x, v_{x}, v_{y}\right) \in \mathbb{R}_{t} \times \Sigma^{-}, x=0 \\ g_{L}, & \left(t, x, v_{x}, v_{y}\right) \in \mathbb{R}_{t} \times \Sigma^{-}, x=L .\end{cases}
$$

We impose Silver-Müller condition on the electro-magnetic field $\left(E_{x}, E_{y}, B_{z}\right)$ :

$$
\begin{gathered}
n_{0} \wedge E+c \cdot n_{0} \wedge\left(n_{0} \wedge B\right)=h_{0}, \\
n_{L} \wedge E+c \cdot n_{L} \wedge\left(n_{L} \wedge B\right)=h_{L},
\end{gathered}
$$

where $n_{0}=(-1,0,0)$ and $n_{L}=(1,0,0)$ are the outward unit normals of $] 0, L[$ in $x=0$ and $x=L$. Here, the boundary data $g_{0}, g_{L}, h_{0}, h_{L}$ are $T$ periodic functions and we look for $T$ periodic solutions $\left(f, E_{x}, E_{y}, B_{z}\right)$ of the $(V M)$ problem (1), (2), (3), (4), (6), (7) and (8). The formulas (7) and (8) model incoming waves in the device and can be written:

$$
\begin{array}{cl}
E_{y}(t, 0)+c B_{z}(t, 0)=h_{0}(t), & t \in \mathbb{R}_{t}, \\
E_{y}(t, L)-c B_{z}(t, L)=h_{L}(t), & t \in \mathbb{R}_{t} .
\end{array}
$$

$\mathrm{RR} \mathrm{n}^{\circ} 3554$ 
Mihai Bostan

One of the key point of our proof of existence of such solutions is to control the life-time of particles in the domain $] 0, L[$. Therefore we impose a non-vanishing condition of incoming velocities which reads :

$$
\begin{gathered}
\operatorname{supp}\left(g_{0}\right) \subset\left\{\left(t, x, v_{x}, v_{y}\right) ; t \in \mathbb{R}_{t}, x=0,0<v_{0} \leq v_{x}, \sqrt{v_{x}^{2}+v_{y}^{2}} \leq v_{1}\right\}, \\
\operatorname{supp}\left(g_{L}\right) \subset\left\{\left(t, x, v_{x}, v_{y}\right) ; t \in \mathbb{R}_{t}, x=L, 0>-v_{0} \geq v_{x}, \sqrt{v_{x}^{2}+v_{y}^{2}} \leq v_{1}\right\},
\end{gathered}
$$

for $0<v_{0}<v_{1}$ given. On the other hand, in order to preserve the periodicity of $E_{x}$ given by (2), a time averaging vanishing condition of the incoming current is imposed:

$$
\int_{0}^{T} d t \int_{v_{x}>0} \int_{v_{y}} v_{x} g_{0}\left(t, v_{x}, v_{y}\right) d v+\int_{0}^{T} d t \int_{v_{x}<0} \int_{v_{y}} v_{x} g_{L}\left(t, v_{x}, v_{y}\right) d v=0 .
$$

Let us remark that even if the electro-magnetic field $\left(E_{x}, E_{y}, B_{z}\right)$ is "a priori" known, there is no uniqueness of the $T$ periodic solution of the Vlasov problem $(V):(1)$ and (6). Indeed, the distribution function can take arbitrary (constant) values on the characteristics which remain in the domain (trapped characteristics). In order to select physical solution we introduce as in [1] and [4] the concept of minimal solution of $(V)$ which are the solutions which vanish on the trapped characteristics. These solutions can be obtained as the limit of the ( unique ) solution of the modified Vlasov problem $\left(V_{\alpha}\right)$ when an absorption term $\alpha>0$ is introduced and tends to zero :

$$
\begin{aligned}
\alpha f+\partial_{t} f+v_{x} \cdot \partial_{x} f+\frac{q}{m}\left(E_{x}+v_{y} \cdot B_{z}\right) \cdot \partial_{v_{x}} f & +\frac{q}{m}\left(E_{y}-v_{x} \cdot B_{z}\right) \cdot \partial_{v_{y}} f=0 \\
\left(t, x, v_{x}, v_{y}\right) & \left.\in \mathbb{R}_{t} \times\right] 0, L\left[\times \mathbb{R}_{v}^{2} .\right.
\end{aligned}
$$

This limit absorption principle has been developped by the author to obtain numerical periodic solutions of Partial Differential Equation, see [13]. We also stress that these results has been announced in [11].

The paper is organized as followed. In Section 2 we define weak solutions and minimal mild solution of the Vlasov problem $(V)$. We also proved that the weak solution of the modified Vlasov problem $\left(V_{\alpha}\right)$ is unique and coincide with 
the minimal mild solution. In section 3 we prove existence of weak periodic solution for the classical 1D Vlasov-Maxwell system. We introduce a regularized problem. The existence theorem is obtained by using Schauder's theorem for the modified problem. Then we pass to the limit in the regularization parameter to obtain our main result. Section 4 is devoted to the relativistic 1D Vlasov-Maxwell system.

\section{Definitions and bounds for the Vlasov equa- tion}

In this section we assume that the electro-magnetic field $\left(E_{x}, E_{y}, B_{z}\right)$ is $T$ periodic in time and we look for a solution $f$ of the Vlasov equation:

$$
\begin{aligned}
& \partial_{t} f+v_{x} \cdot \partial_{x} f+\frac{q}{m}\left(E_{x}+v_{y} \cdot B_{z}\right) \cdot \partial_{v_{x}} f+\frac{q}{m}\left(E_{y}-v_{x} \cdot B_{z}\right) \cdot \partial_{v_{y}} f=0, \\
& \left.\left(t, x, v_{x}, v_{y}\right) \in \mathbb{R}_{t} \times\right] 0, L\left[\times \mathbb{R}_{v}^{2},\right. \\
& f\left(t, 0, v_{x}, v_{y}\right)=g_{0}\left(t, v_{x}, v_{y}\right), \quad t \in \mathbb{R}_{t} v_{x}>0, v_{y} \in \mathbb{R}_{v} \\
& f\left(t, L, v_{x}, v_{y}\right)=g_{L}\left(t, v_{x}, v_{y}\right), \quad t \in \mathbb{R}_{t} v_{x}<0, v_{y} \in \mathbb{R}_{v} .
\end{aligned}
$$

Here $q(>0)$ and $m$ are the charge and the mass of particles. Moreover, we suppose that the given distribution functions $g_{0}, g_{L}$ of the in-flowing particles are $T$ periodic in time, too. Now we briefly recall the notions of mild and weak solutions for this type of problem.

\subsection{Weak and mild solution of the Vlasov equation}

Definition 1 Let $E_{x}, E_{y}, B_{z} \in L^{\infty}\left(\mathbb{R}_{t} \times\right] 0, L[)$ and $g_{0}, g_{L} \in L_{l o c}^{1}\left(\mathbb{R}_{t} \times \Sigma^{-}\right)$be $T$ periodic functions in time, where:

$$
\begin{aligned}
\Sigma^{-} & =\left\{\left(t, x, v_{x}, v_{y}\right) \mid t \in \mathbb{R}, x=0, v_{x}>0, v_{y} \in \mathbb{R}\right\} \\
& \cup\left\{\left(t, x, v_{x}, v_{y}\right) \mid t \in \mathbb{R}, x=L, v_{x}<0, v_{y} \in \mathbb{R}\right\} .
\end{aligned}
$$

$\mathrm{RR} \mathrm{n}^{\circ} 3554$ 
We say that $f \in L_{\text {loc }}^{1}\left(\mathbb{R}_{t} \times\right] 0, L\left[\times \mathbb{R}_{v}^{2}\right)$ is a $T$ periodic weak solution of problem (14), (15), (16) iff:

$$
\begin{aligned}
\int_{0}^{T} \int_{0}^{L} \int_{\mathbb{R}_{v}^{2}}\left(\partial_{t} \theta+v_{x} \cdot \partial_{x} \theta\right. & +\frac{q}{m}\left(E_{x}+v_{y} \cdot B_{z}\right) \cdot \partial_{v_{x}} \theta \\
& \left.+\frac{q}{m}\left(E_{y}-v_{x} \cdot B_{z}\right) \cdot \partial_{v_{y}} \theta\right) f\left(t, x, v_{x}, v_{y}\right) d v d x d t \\
& =\int_{0}^{T} \int_{v_{x}<0} \int_{v_{y}} v_{x} g_{L}\left(t, v_{x}, v_{y}\right) \theta\left(t, L, v_{x}, v_{y}\right) d v d t \\
& -\int_{0}^{T} \int_{v_{x}>0} \int_{v_{y}} v_{x} g_{0}\left(t, v_{x}, v_{y}\right) \theta\left(t, 0, v_{x}, v_{y}\right) d v d t
\end{aligned}
$$

for all $T$ periodic function $\theta \in \mathcal{V}$, where:

$$
\begin{array}{r}
\mathcal{V}=\left\{\eta \in W^{1, \infty}\left(\mathbb{R}_{t} \times\right] 0, L\left[\times \mathbb{R}_{v}^{2}\right) ; \eta\left(t, 0, v_{x}<0, v_{y}\right)=\eta\left(t, L, v_{x}>0, v_{y}\right)=0,\right. \\
\left.\operatorname{supp}(\eta) \text { bounded set of } \mathbb{R}_{t} \times[0, L] \times \mathbb{R}_{v}^{2}\right\}
\end{array}
$$

In other words, a weak solution of problem (14), (15), (16) is a distribution function satisfying:

$$
\begin{aligned}
<f, \varphi> & =\int_{0}^{T} \int_{v_{x}<0} \int_{v_{y}} v_{x} \cdot g_{L}\left(t, v_{x}, v_{y}\right) \cdot \theta\left(t, L, v_{x}, v_{y}\right) d v d t \\
& -\int_{0}^{T} \int_{v_{x}>0} \int_{v_{y}} v_{x} \cdot g_{0}\left(t, v_{x}, v_{y}\right) \cdot \theta\left(t, 0, v_{x}, v_{y}\right) d v d t
\end{aligned}
$$

for all $T$ periodic function $\varphi$, where $\theta$ denote the solution of the problem:

$$
\begin{gathered}
\partial_{t} \theta+v_{x} \cdot \partial_{x} \theta+\frac{q}{m}\left(E_{x}+v_{y} \cdot B_{z}\right) \cdot \partial_{v_{x}} \theta+\frac{q}{m}\left(E_{y}-v_{x} \cdot B_{z}\right) \cdot \partial_{v_{y}} \theta=\varphi \\
\left.\left(t, x, v_{x}, v_{y}\right) \in \mathbb{R}_{t} \times\right] 0, L\left[\times \mathbb{R}_{v}^{2}\right. \\
\theta\left(t, 0, v_{x}, v_{y}\right)=0, \quad t \in \mathbb{R}_{t} v_{x}<0, v_{y} \in \mathbb{R}_{v} \\
\theta\left(t, L, v_{x}, v_{y}\right)=0, \quad t \in \mathbb{R}_{t} v_{x}>0, v_{y} \in \mathbb{R}_{v}
\end{gathered}
$$


Remark 1 In the above definition we can assume that the electro-magnetic field is only in $\left(L^{p}\left(\mathbb{R}_{t} \times\right] 0, L[)\right)^{3}$ by requiring more regularity on $f$ (and $g_{0}, g_{L}$ ), namely $f$ in $L_{\text {loc }}^{q}\left(\mathbb{R}_{t} \times\right] 0, L\left[\times \mathbb{R}_{v}^{2}\right)$ where $q$ is the conjugate exponent.

If the electro-magnetic field satisfies $\left(E_{x}, E_{y}, B_{z}\right) \in\left(L^{\infty}\left(\mathbb{R}_{t} ; W^{1, \infty}(] 0, L[)\right)\right)^{3}$, we can express a solution in terms of characteristics. Let $\left(t, x, v_{x}, v_{y}\right)$ belong to $\left.\mathbb{R}_{t} \times\right] 0, L\left[\times \mathbb{R}_{v}^{2}\right.$, we denote by $X\left(s ; x, v_{x}, v_{y}, t\right), \quad V_{x}\left(s ; x, v_{x}, v_{y}, t\right)$ and $V_{y}\left(s ; x, v_{x}, v_{y}, t\right)$ the solution of the system:

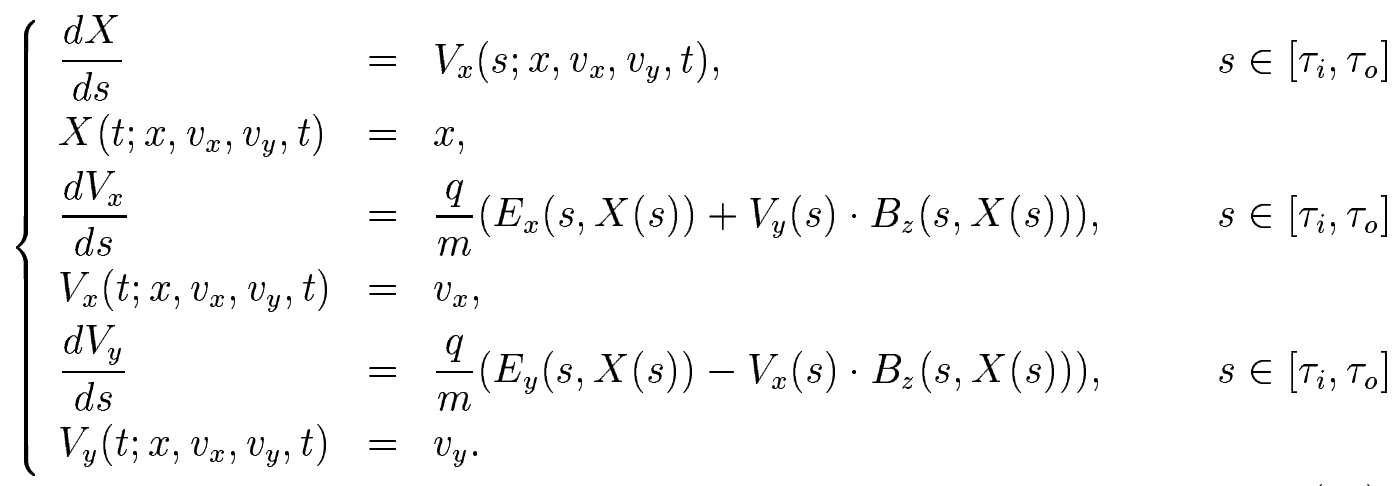

where $\left[\tau_{i}\left(x, v_{x}, v_{y}, t\right), \tau_{o}\left(x, v_{x}, v_{y}, t\right)\right]$ is the life-time of the particle in the domain ] $0, L[$ :

$$
\left(X\left(\tau_{i}\right), V_{x}\left(\tau_{i}\right), V_{y}\left(\tau_{i}\right)\right) \in \Sigma^{-}
$$

and

$$
\left(X\left(\tau_{o}\right), V_{x}\left(\tau_{o}\right), V_{y}\left(\tau_{o}\right)\right) \in \Sigma^{+} \cup \Sigma^{0} .
$$

The subsets of $\{0, L\} \times \mathbb{R}_{v}^{2} \Sigma^{+}$and $\Sigma^{0}$ are respectively defined by:

$$
\begin{aligned}
\Sigma^{+}= & \left\{\left(t, x, v_{x}, v_{y}\right) \mid t \in \mathbb{R}, x=0, v_{x}<0, v_{y} \in \mathbb{R}\right\} \\
& \cup\left\{\left(t, x, v_{x}, v_{y}\right) \mid t \in \mathbb{R}, x=L, v_{x}>0, v_{y} \in \mathbb{R}\right\}, \\
\Sigma^{0} & =\left\{\left(t, x, v_{x}, v_{y}\right) \mid t \in \mathbb{R}, x=0, v_{x}=0, v_{y} \in \mathbb{R}\right\} \\
& \cup\left\{\left(t, x, v_{x}, v_{y}\right) \mid t \in \mathbb{R}, x=L, v_{x}=0, v_{y} \in \mathbb{R}\right\} .
\end{aligned}
$$

Using the Cauchy-Lipschitz theorem, we notice that the characteristics are well defined. By integration along the characteristics curves, the solution of the problem (20), (21),(22) writes:

$\mathrm{RR} \mathrm{n}^{\circ} 3554$ 
$\theta\left(t, x, v_{x}, v_{y}\right)=-\int_{t}^{\tau_{o}} \varphi\left(s, X\left(s ; x, v_{x}, v_{y}, t\right), V_{x}\left(s ; x, v_{x}, v_{y}, t\right), V_{y}\left(s ; x, v_{x}, v_{y}, t\right) d s\right.$

Now, (19) implies that:

$$
\begin{aligned}
<f, \varphi>= & \int_{0}^{T} d t \int_{v_{x}>0} \int_{v_{y}} d v \int_{t}^{\tau_{o}} v_{x} \cdot g_{0}\left(t, v_{x}, v_{y}\right) \\
& \varphi\left(s, X\left(s ; 0, v_{x}, v_{y}, t\right), V_{x}\left(s ; 0, v_{x}, v_{y}, t\right), V_{y}\left(s ; 0, v_{x}, v_{y}, t\right) d s\right. \\
- & \int_{0}^{T} d t \int_{v_{x}<0} \int_{v_{y}} d v \int_{t}^{\tau_{o}} v_{x} \cdot g_{L}\left(t, v_{x}, v_{y}\right) \\
& \varphi\left(s, X\left(s ; L, v_{x}, v_{y}, t\right), V_{x}\left(s ; L, v_{x}, v_{y}, t\right), V_{y}\left(s ; L, v_{x}, v_{y}, t\right) d s\right.
\end{aligned}
$$

which is equivalent to:

$$
f\left(t, x, v_{x}, v_{y}\right)=\left\{\begin{aligned}
g_{0}\left(\tau_{i}, V_{x}\left(\tau_{i} ; x,\right.\right. & \left.\left.v_{x}, v_{y}, t\right), V_{y}\left(\tau_{i} ; x, v_{x}, v_{y}, t\right)\right) \\
& \text { if } \tau_{i}>-\infty \text { and } X\left(\tau_{i} ; x, v_{x}, v_{y}, t\right)=0, \\
g_{L}\left(\tau_{i}, V_{x}\left(\tau_{i} ; x,\right.\right. & \left.\left.v_{x}, v_{y}, t\right), V_{y}\left(\tau_{i} ; x, v_{x}, v_{y}, t\right)\right) \\
& \text { if } \tau_{i}>-\infty \text { and } X\left(\tau_{i} ; x, v_{x}, v_{y}, t\right)=L, \\
0 . & \text { otherwise. }
\end{aligned}\right.
$$

Definition 2 Let $E_{x}, E_{y}, B_{z} \in L^{\infty}\left(\mathbb{R}_{t} ; W^{1, \infty}(] 0, L[)\right)$ and $g_{0}, g_{L} \in L_{l o c}^{1}\left(\mathbb{R}_{t} \times\right.$ $\left.\Sigma^{-}\right)$be $T$ periodic functions. The function $f \in L_{\text {loc }}^{1}\left(\mathbb{R}_{t} \times\right] 0, L\left[\times \mathbb{R}_{v}^{2}\right)$ which is the mild periodic solution of problem (14), (15), (16) is given by (26). 
Remark 2 There is in general no uniqueness of the weak solution because $f$ can take arbitrarily values on the characteristics such that $\tau_{i}=-\infty$. But it is possible to prove that the mild solution is the unique minimal solution of the transport equation. We refer to $[P, V M]$ for the concept of the minimal solution and to [Bod, PhD] for a proof of this assertion.

Remark 3 We have that $X\left(s+T ; x, v_{x}, v_{y}, t+T\right)=X\left(s ; x, v_{x}, v_{y}, t\right), V_{x}(s+$ $\left.T ; x, v_{x}, v_{y}, t+T\right)=V_{x}\left(s ; x, v_{x}, v_{y}, t\right), V_{y}\left(s+T ; x, v_{x}, v_{y}, t+T\right)=V_{y}\left(s ; x, v_{x}, v_{y}, t\right)$ and $\tau_{i}\left(x, v_{x}, v_{y}, t+T\right)=\tau_{i}\left(x, v_{x}, v_{y}, t\right)+T$ because of the periodicity of $E_{x}, E_{y}, B_{z}$. Using this equality it is easy to check that the mild solution is periodic.

Remark 4 If $g_{0}, g_{L} \in C^{1}\left(\mathbb{R}_{t} \times \Sigma^{-}\right)$then the mild solution is a classical solution of (14), (15), (16).

\subsection{Estimation of the life-time of particles}

In order to assure $L^{\infty}$ estimates for the charge and current densities, we assume that the following conditions are satisfied:

$$
\begin{gathered}
\|E\|_{L^{\infty}}+\left\|B_{z}\right\|_{L^{\infty}} \cdot\left(v_{1}+\frac{q}{m}\|E\|_{L^{\infty}} \frac{2 L}{v_{0}}\right) \leq \frac{m v_{0}^{2}}{4 q L} \\
\left(E_{x}, E_{y}, B_{z}\right) \in\left(L^{\infty}\left(\mathbb{R}_{t} ; W^{1, \infty}(] 0, L[)\right)\right)^{3}, \\
\operatorname{supp}\left(g_{0}\right) \subset\left\{\left(t, x, v_{x}, v_{y}\right) ; t \in \mathbb{R}_{t}, x=0,0<v_{0} \leq v_{x}, \sqrt{v_{x}^{2}+v_{y}^{2}} \leq v_{1}\right\}, \\
\operatorname{supp}\left(g_{L}\right) \subset\left\{\left(t, x, v_{x}, v_{y}\right) ; t \in \mathbb{R}_{t}, x=L, 0>-v_{0} \geq v_{x}, \sqrt{v_{x}^{2}+v_{y}^{2}} \leq v_{1}\right\} .
\end{gathered}
$$

Here, $|E|_{L^{\infty}}$ is the $L^{\infty}$ norm of $\sqrt{E_{x}^{2}+E_{y}^{2}}$ and $v_{0}, v_{1}$ are constants which will be chosen in the next section. With these assumptions, we get:

$\mathrm{RR} \mathrm{n}^{\circ} 3554$ 
Lemma 1 Assume that the electro-magnetic field and the boundary data satisfy (28),(29) and (30). Then, the life-time in $] 0, L[$ of particles starting from the support of $g_{0}$ and $g_{L}$ is finite:

$$
\tau_{o}\left(x, v_{x}, v_{y}, t\right)-\tau_{i}\left(x, v_{x}, v_{y}, t\right) \leq 2 \cdot \frac{L}{v_{0}}, \forall\left(t, x, v_{x}, v_{y}\right) \in \operatorname{supp}\left(g_{0}\right) \cup \operatorname{supp}\left(g_{L}\right)
$$

Proof Suppose that there is a particle starting from the support of $g_{0}$ at $t=\tau_{i}$ and which is still in $] 0, L\left[\right.$ at $\tau_{i}+\frac{2 L}{v_{0}}<\tau_{o}$ :

$$
\left.X\left(\tau_{i}+\frac{2 L}{v_{0}}\right) \in\right] 0, L[
$$

According to (30), we have:

$$
\begin{gathered}
0<v_{0} \leq V_{x}\left(\tau_{i}\right) \\
\sqrt{V_{x}^{2}\left(\tau_{i}\right)+V_{y}^{2}\left(\tau_{i}\right)} \leq v_{1} .
\end{gathered}
$$

We multiply the velocity equations of $(23)$ by $V_{x}(s)$ and $V_{y}(s)$ to get for $s \in$ $\left[\tau_{i}, \tau_{o}\right]:$

$$
\begin{aligned}
& \frac{1}{2} \cdot \frac{d}{d s}\left|V_{x}(s)\right|^{2}=\frac{q}{m}\left(E_{x}(s, X(s)) \cdot V_{x}(s)+V_{x}(s) \cdot V_{y}(s) \cdot B_{z}(s, X(s))\right), \\
& \frac{1}{2} \cdot \frac{d}{d s}\left|V_{y}(s)\right|^{2}=\frac{q}{m}\left(E_{y}(s, X(s)) \cdot V_{y}(s)-V_{y}(s) \cdot V_{x}(s) \cdot B_{z}(s, X(s))\right),
\end{aligned}
$$

and therefore:

$$
\frac{d}{d s} \sqrt{V_{x}^{2}(s)+V_{y}^{2}(s)} \leq \frac{q}{m} \sqrt{E_{x}^{2}(s, X(s))+E_{y}^{2}(s, X(s))}
$$


which yields:

$$
\begin{aligned}
\left|\sqrt{V_{x}^{2}(s)+V_{y}^{2}(s)}-\sqrt{v_{x}^{2}+v_{y}^{2}}\right| & \leq \frac{q}{m} \cdot\left\|\sqrt{E_{x}^{2}+E_{y}^{2}}\right\|_{L^{\infty}} \cdot\left(s-\tau_{i}\right) \\
& \leq \frac{q}{m} \cdot\left\|\sqrt{E_{x}^{2}+E_{y}^{2}}\right\|_{L^{\infty}} \cdot \frac{2 L}{v_{0}} .
\end{aligned}
$$

Integrating (23) on $\left[\tau_{i}, t\right] \subset\left[\tau_{i}, \tau_{i}+2 L / v_{0}\right]$, we obtain:

$$
\begin{gathered}
X(t)=X\left(\tau_{i}\right)+\int_{\tau_{i}}^{t} V_{x}(s) d s \\
V_{x}(t)=V_{x}\left(\tau_{i}\right)+\int_{\tau_{i}}^{t} \frac{q}{m}\left(E_{x}(s)+V_{y}(s) \cdot B_{z}(s)\right) d s, \\
V_{y}(t)=V_{y}\left(\tau_{i}\right)+\int_{\tau_{i}}^{t} \frac{q}{m}\left(E_{y}(s)-V_{x}(s) \cdot B_{z}(s)\right) d s .
\end{gathered}
$$

From (35) and (34) we deduce that for all $s \in\left[\tau_{i}, \tau_{i}+2 L / v_{0}\right]$ :

$$
\left|V_{y}(s)\right| \leq v_{1}+\left(s-\tau_{i}\right) \frac{q}{m}\|E\|_{L^{\infty}} .
$$

Now using (33), (37), (39) and (28) we find for all $t \in\left[\tau_{i}, \tau_{i}+2 L / v_{0}\right]$ :

$$
\begin{aligned}
V_{x}(t) & \geq v_{x}-\int_{\tau_{i}}^{t} \frac{q}{m} \cdot\left(\left|E_{x}(s)\right|+\left|B_{z}(s)\right| \cdot\left|V_{y}(s)\right|\right) d s \\
& \geq v_{0}-\int_{\tau_{i}}^{t} \frac{q}{m} \cdot\left(\left\|E_{x}\right\|_{L^{\infty}}+\left\|B_{z}\right\|_{L^{\infty}} \cdot\left(v_{1}+\left(s-\tau_{i}\right) \frac{q}{m}\|E\|_{L^{\infty}}\right)\right) d s \\
& >v_{0}-\frac{2 L}{v_{0}} \cdot \frac{q}{m} \cdot\left(\|E\|_{L^{\infty}}+\left\|B_{z}\right\|_{L^{\infty}} \cdot\left(v_{1}+\frac{q}{m}\|E\|_{L^{\infty}} \frac{2 L}{v_{0}}\right)\right) \\
& \geq v_{0}-\frac{v_{0}}{2}=\frac{v_{0}}{2} .
\end{aligned}
$$

Now, from (94) we deduce:

$$
\begin{aligned}
X\left(\tau_{i}+2 L / v_{0}\right) & =0+\int_{\tau_{i}}^{\tau_{i}+2 L / v_{0}} V_{x}(s) d s \\
& >\frac{2 L}{v_{0}} \cdot \frac{v_{0}}{2}=L
\end{aligned}
$$

$\mathrm{RR} \mathrm{n}^{\circ} 3554$ 
which contradicts (32). If the particle starts from the support of $g_{L}$, using the same ideas as previous we prove that $\tau_{o} \leq \tau_{i}+\frac{2 L}{v_{0}}$ also holds.

Corollary 1 Assuming the same hypotheses as in Lemma 1 and let $f$ be the mild solution of Definition 2. Then we have:

$$
\begin{gathered}
\operatorname{supp}(f) \subset\left\{\left(t, x, v_{x}, v_{y}\right)\left|t \in \mathbb{R}_{t}, x \in[0, L], \frac{v_{0}}{2} \leq\right| v_{x} \mid, \sqrt{v_{x}^{2}+v_{y}^{2}} \leq v_{1}+\frac{v_{0}}{2}\right\} \\
\|\rho\|_{L^{\infty}} \leq \frac{\pi}{2}\left(v_{1}+v_{0} / 2\right)^{2} \cdot q \cdot\left(\left\|g_{0}\right\|_{L^{\infty}}+\left\|g_{L}\right\|_{L^{\infty}}\right),
\end{gathered}
$$

and

$$
\max \left\{\left\|j_{x}\right\|_{L^{\infty}},\left\|j_{y}\right\|_{L^{\infty}}\right\} \leq \frac{\pi}{2}\left(v_{1}+v_{0} / 2\right)^{3} \cdot q \cdot\left(\left\|g_{0}\right\|_{L^{\infty}}+\left\|g_{L}\right\|_{L^{\infty}}\right),
$$

where $\rho(t, x)=q \int_{\mathbb{R}_{v}^{2}} f\left(t, x, v_{x}, v_{y}\right) d v$ and $j_{x, y}(t, x)=q \int_{\mathbb{R}_{v}^{2}} v_{x, y} \cdot f\left(t, x, v_{x}, v_{y}\right) d v$. Proof The estimate (42) follow from the previous Lemma. Indeed, according to (35), (30) and (28), we obtain:

$$
\begin{aligned}
\sqrt{V_{x}^{2}(t)+V_{y}^{2}(t)} & \leq \sqrt{V_{x}^{2}\left(\tau_{i}\right)+V_{y}^{2}\left(\tau_{i}\right)}+\int_{\tau_{i}}^{t} \frac{q}{m}|E(s, X(s))| d s \\
& \leq v_{1}+\left(t-\tau_{i}\right) \cdot \frac{q}{m}|E|_{L^{\infty}} \\
& \leq v_{1}+\frac{2 L q}{m v_{0}}|E|_{L^{\infty}} \\
& \leq v_{1}+\frac{v_{0}}{2} .
\end{aligned}
$$

Using (37) and (30) we get for $t \in\left[\tau_{i}, \tau_{o}\right]$ :

$$
\begin{aligned}
V_{x}(t) & \geq V_{x}\left(\tau_{i}\right)-\int_{\tau_{i}}^{t} \frac{q}{m}\left(\left|E_{x}(s, X(s))\right|+\left|V_{y}(s) \| B_{z}(s, X(s))\right|\right) d s \\
& \geq v_{0}-\left(\tau_{o}-\tau_{i}\right) \cdot \frac{q}{m} \cdot\left(\|E\|_{L^{\infty}}+\left\|B_{z}\right\|_{L^{\infty}} \cdot\left(v_{1}+\frac{q}{m}\|E\|_{L^{\infty}} \frac{2 L}{v_{0}}\right)\right) \\
& \geq v_{0}-\frac{2 L}{v_{0}} \cdot \frac{q}{m} \cdot\left(\|E\|_{L^{\infty}}+\left\|B_{z}\right\|_{L^{\infty}} \cdot\left(v_{1}+\frac{q}{m}\|E\|_{L^{\infty}} \frac{2 L}{v_{0}}\right)\right) \\
& \geq v_{0}-\frac{v_{0}}{2}=\frac{v_{0}}{2} .
\end{aligned}
$$


If the particle starts from the support of $g_{L},(45)$ are the same and (46) change in:

$$
\begin{aligned}
-V_{x}(t) & \geq-V_{x}\left(\tau_{i}\right)-\int_{\tau_{i}}^{t} \frac{q}{m}\left(\left|E_{x}(s, X(s))\right|+\left|V_{y}(s)\right|\left|B_{z}(s, X(s))\right|\right) d s \\
& \geq v_{0}-\frac{v_{0}}{2}=\frac{v_{0}}{2} .
\end{aligned}
$$

Now, (43) and (44) can be easily checked. For $\left.(t, x) \in \mathbb{R}_{t} \times\right] 0, L[$ we have:

$$
\begin{aligned}
\rho(t, x) & =q \int_{\mathbb{R}_{v^{2}}} f\left(t, x, v_{x}, v_{y}\right) d v \\
& =q \int_{v_{x}>0} \int_{\mathbb{R}_{v}} f\left(t, x, v_{x}, v_{y}\right) d v+q \int_{v_{x}<0} \int_{\mathbb{R}_{v}} f\left(t, x, v_{x}, v_{y}\right) d v \\
& \leq \frac{\pi}{2}\left(v_{1}+v_{0} / 2\right)^{2} \cdot q \cdot\left(\left\|g_{0}\right\|_{L^{\infty}}+\left\|g_{L}\right\|_{L^{\infty}}\right)
\end{aligned}
$$

and therefore $\|\rho\|_{L^{\infty}} \leq \frac{\pi}{2}\left(v_{1}+v_{0} / 2\right)^{2} \cdot q \cdot\left(\left\|g_{0}\right\|_{L^{\infty}}+\left\|g_{L}\right\|_{L^{\infty}}\right)$. Obviously, (44) follows in the same way.

Remark 5 Assuming the same hypotheses as in Lemma 1. Then the mild solution $f$ of Definition 2 can be split in two mild solutions $f=f_{0}+f_{L}$ given by:

$$
\begin{aligned}
\left\langle f_{0}, \varphi>=\right. & \int_{0}^{T} d t \int_{v_{x}>0} \int_{v_{y}} d v \int_{t}^{\tau_{o}} v_{x} \cdot g_{0}\left(t, v_{x}, v_{y}\right) \\
& \cdot \quad \varphi\left(s, X\left(s ; 0, v_{x}, v_{y}, t\right), V_{x}\left(s ; 0, v_{x}, v_{y}, t\right), V_{y}\left(s ; 0, v_{x}, v_{y}, t\right) d s\right.
\end{aligned}
$$

and:

$$
\begin{aligned}
\left\langle f_{L}, \varphi>=\right. & -\int_{0}^{T} d t \int_{v_{x}<0} \int_{v_{y}} d v \int_{t}^{\tau_{o}} v_{x} \cdot g_{L}\left(t, v_{x}, v_{y}\right) \\
& \quad \varphi\left(s, X\left(s ; L, v_{x}, v_{y}, t\right), V_{x}\left(s ; L, v_{x}, v_{y}, t\right), V_{y}\left(s ; L, v_{x}, v_{y}, t\right) d s .\right.
\end{aligned}
$$

$\mathrm{RR} \mathrm{n}^{\circ} 3554$ 
In the same time $f_{0}, f_{L}$ are weak periodic solutions for the problems:

$$
\begin{gathered}
\partial_{t} f_{0}+v_{x} \cdot \partial_{x} f_{0}+\frac{q}{m}\left(E_{x}+v_{y} \cdot B_{z}\right) \cdot \partial_{v_{x}} f_{0} \\
\left(t, x, v_{x}, v_{y}\right) \quad \frac{q}{m}\left(E_{y}-v_{x} \cdot B_{z}\right) \cdot \partial_{v_{y}} f_{0}=0,0, L\left[\times \mathbb{R}_{v}^{2},\right. \\
f_{0}\left(t, 0, v_{x}, v_{y}\right)=g_{0}\left(t, v_{x}, v_{y}\right), \quad t \in \mathbb{R}_{t} v_{x}>0, v_{y} \in \mathbb{R}_{v}, \\
f_{0}\left(t, L, v_{x}, v_{y}\right)=0, \quad t \in \mathbb{R}_{t} v_{x}<0, v_{y} \in \mathbb{R}_{v},
\end{gathered}
$$

and:

$$
\begin{gathered}
\partial_{t} f_{L}+v_{x} \cdot \partial_{x} f_{L}+\frac{q}{m}\left(E_{x}+v_{y} \cdot B_{z}\right) \cdot \partial_{v_{x}} f_{L} \\
\left.\left(t, x, v_{x}, v_{y}\right) \in \frac{q}{m}\left(E_{y}-v_{x} \cdot B_{z}\right) \cdot \partial_{v_{y}} f_{L}=0\right] 0, L\left[\times \mathbb{R}_{v}^{2}\right. \\
f_{L}\left(t, 0, v_{x}, v_{y}\right)=0, \quad t \in \mathbb{R}_{t} v_{x}>0, v_{y} \in \mathbb{R}_{v} \\
f_{L}\left(t, L, v_{x}, v_{y}\right)=g_{L}\left(t, v_{x}, v_{y}\right), \quad t \in \mathbb{R}_{t} v_{x}<0, v_{y} \in \mathbb{R}_{v} .
\end{gathered}
$$

Moreover we have :

$$
\operatorname{supp}\left(f_{0}\right) \subset\left\{\left(t, x, v_{x}, v_{y}\right) \mid t \in \mathbb{R}_{t}, x \in[0, L], \frac{v_{0}}{2} \leq v_{x}, \sqrt{v_{x}^{2}+v_{y}^{2}} \leq v_{1}+\frac{v_{0}}{2}\right\},
$$

and :

$$
\operatorname{supp}\left(f_{L}\right) \subset\left\{\left(t, x, v_{x}, v_{y}\right) \mid t \in \mathbb{R}_{t}, x \in[0, L],-\frac{v_{0}}{2} \geq v_{x}, \sqrt{v_{x}^{2}+v_{y}^{2}} \leq v_{1}+\frac{v_{0}}{2}\right\} .
$$




\section{Weak periodic solutions for the modified 1D Vlasov-Maxwell system.}

Our goal is to establish existence result for the weak periodic solution of the 1D Vlasov-Maxwell problem:

$$
\begin{gathered}
\partial_{t} f+v_{x} \cdot \partial_{x} f+\frac{q}{m}\left(E_{x}+v_{y} \cdot B_{z}\right) \cdot \partial_{v_{x}} f+\frac{q}{m}\left(E_{y}-v_{x} \cdot B_{z}\right) \cdot \partial_{v_{y}} f=0, \\
\left.\left(t, x, v_{x}, v_{y}\right) \in \mathbb{R}_{t} \times\right] 0, L\left[\times \mathbb{R}_{v}^{2},\right. \\
\left.\partial_{t} E_{x}=-\frac{1}{\varepsilon} j_{x}:=-\frac{1}{\varepsilon} \int_{v} v_{x} f\left(t, x, v_{x}, v_{y}\right) d v, \quad(t, x) \in \mathbb{R}_{t} \times\right] 0, L[, \\
\left.\partial_{t} E_{y}+c^{2} \partial_{x} B_{z}=-\frac{1}{\varepsilon} j_{y}:=-\frac{1}{\varepsilon} \int_{v} v_{y} f\left(t, x, v_{x}, v_{y}\right) d v, \quad(t, x) \in \mathbb{R}_{t} \times\right] 0, L[, \\
\partial_{t} B_{z}+\partial_{x} E_{y}=0,
\end{gathered}
$$

with the boundary conditions:

$$
\begin{gathered}
f\left(t, 0, v_{x}, v_{y}\right)=g_{0}\left(t, v_{x}, v_{y}\right), \quad t \in \mathbb{R}_{t} v_{x}>0, v_{y} \in \mathbb{R}_{v}, \\
f\left(t, L, v_{x}, v_{y}\right)=g_{L}\left(t, v_{x}, v_{y}\right), \quad t \in \mathbb{R}_{t} v_{x}<0, v_{y} \in \mathbb{R}_{v}, \\
E_{y}(t, 0)+c B_{z}(t, 0)=h_{0}(t), \quad t \in \mathbb{R}_{t}, \\
E_{y}(t, L)-c B_{z}(t, L)=h_{L}(t), \quad t \in \mathbb{R}_{t},
\end{gathered}
$$

$\mathrm{RR} \mathrm{n}^{\circ} 3554$ 
Here, the boundary data $g_{0}, g_{L}, h_{0}, h_{L}$ are $T$ - periodic functions and $c$ is the light velocity in the vacuum. We look for a weak periodic solution $\left(f\left(t, x, v_{x}, v_{y}\right)\right.$, $\left.E_{x}(t, x), E_{y}(t, x), B_{z}(t, x)\right)$. The Schauder fixed point theorem is used. We define an application which maps a periodic electro-magnetic field $\left(E_{x}, E_{y}, B_{z}\right)$ to an other one $\left(E_{x}^{1}, E_{y}^{1}, B_{z}^{1}\right)$ where $\left(E_{x}^{1}, E_{y}^{1}, B_{z}^{1}\right)$ is defined as follows. Let $f$ be the mild periodic solution of Definition 2 corresponding to the electromagnetic field $\left(E_{x}, E_{y}, B_{z}\right)$. The new electro-magnetic field $\left(E_{x}^{1}, E_{y}^{1}, B_{z}^{1}\right)$ is determined as the solution of the Maxwell problem with the current density $j_{x, y}(t, x)=\int_{\mathbb{R}_{v}^{2}} v_{x, y} f\left(t, x, v_{x}, v_{y}\right) d v$. Unfortunately this procedure cannot be used directly. Indeed the Definiton 2 requires that the electro-magnetic field is Lipschitz with respect to $x$ and we cannot expect such a regularity in the general case. Therefore we have to regularize the field. We also have to use an absorption term in the Vlasov equation in order to have uniqueness of the weak solution. Then the strategy of proof is as follows. We first show the existence of weak periodic solution for the regularized problem by using the Schauder fixed

point theorem. Next we pass to the limit when the regularization parameter vanishes.

\subsection{Fixed point for the regularized problem}

Let $\mathcal{X}$ be the set of fields $\left(E_{x}, E_{y}, B_{z}\right)$ which verify:

$$
\begin{array}{r}
\mathcal{X}=\left\{\left(E_{x}, E_{y}, B_{z}\right) \in\left(L^{\infty}\left(\mathbb{R}_{t} \times\right] 0, L[)\right)^{3} ;\|E\|_{L^{\infty}} \leq K, c \cdot\left\|B_{z}\right\|_{L^{\infty}} \leq K,\right. \\
\left.\left(E_{x}, E_{y}, B_{z}\right)(t)=\left(E_{x}, E_{y}, B_{z}\right)(t+T) \forall t \in \mathbb{R}_{t}\right\}
\end{array}
$$

where $K$ is a positive constant. Because of time periodicity, $\mathcal{X}$ is a compact set of $\left(L_{T}^{2}\left(\mathbb{R}_{t} \times\right] 0, L[)\right)^{3}$ with the weak topology, where:

$L_{T}^{2}\left(\mathbb{R}_{t} \times\right] 0, L[)=\left\{u ; \int_{0}^{T} \int_{0}^{L}|u(t, x)|^{2} d x d t<\infty, u(t, \cdot)=u(t+T, \cdot) \forall t \in \mathbb{R}_{t}\right\}$

We now introduce a regularization mapping:

$$
R_{\alpha}: L^{\infty}\left(\mathbb{R}_{t} \times\right] 0, L[) \rightarrow L^{\infty}\left(\mathbb{R}_{t} ; C^{1}([0, L]),\right.
$$


$\left(R_{\alpha} E_{x}, R_{\alpha} E_{y}, R_{\alpha} B_{z}\right)(t, x)=\int_{-\infty}^{\infty} \int_{0}^{L} \zeta_{\alpha}(t-s, x-y) \cdot\left(E_{x}, E_{y}, B_{z}\right)(s, y) d s d y$,

where $\zeta_{\alpha} \geq 0$ is a mollifier:

$$
\begin{array}{cc}
\zeta_{\alpha}(t, x)=\frac{1}{\alpha^{2}} \zeta\left(\frac{t}{\alpha}, \frac{x}{\alpha}\right) & , \quad \zeta \in C_{0}^{\infty}\left(\mathbb{R}^{2}\right) \\
\operatorname{supp}(\zeta) \subset[-1,1] \times[-1,1] \quad, \quad \int_{-\infty}^{\infty} \int_{-\infty}^{\infty} \zeta(u, v) d u d v=1
\end{array}
$$

It is easy to see that $\left(R_{\alpha} E_{x}, R_{\alpha} E_{y}, R_{\alpha} B_{z}\right)$ are also time periodic :

$\left(R_{\alpha} E_{x}, R_{\alpha} E_{y}, R_{\alpha} B_{z}\right)(t, x)=\int_{-\infty}^{\infty} \int_{0}^{L} \zeta_{\alpha}(s, x-y) \cdot\left(E_{x}, E_{y}, B_{z}\right)(t-s, y) d s d y$,

and therefore $R_{\alpha}(\mathcal{X}) \subset \mathcal{X}$. Next, we consider the application:

$$
F:\left(E_{x}, E_{y}, B_{z}\right) \in \mathcal{X} \mapsto\left(E_{x}^{1}, E_{y}^{1}, B_{z}^{1}\right),
$$

where:

$$
\begin{aligned}
E_{x}^{1}(t, x)= & -\frac{1}{\varepsilon} \int_{0}^{t}\left(j_{x, \alpha}(s, x)+\alpha \int_{0}^{x} \rho_{0, \alpha}(s, y) d y-\alpha \int_{x}^{L} \rho_{L, \alpha}(s, y) d y\right) d s \\
& \left.+\frac{1}{\varepsilon} \int_{0}^{x} \rho(0, y) d y, \quad(t, x) \in \mathbb{R}_{t} \times\right] 0, L[ \\
E_{y}^{1}(t, x) & =\frac{1}{2}\left(h_{0}(t-x / c)+h_{L}(t-(L-x) / c)\right) \\
& -\frac{1}{2 \varepsilon} \int_{t-x / c}^{t} j_{y, \alpha}(s, x-c(t-s)) d s \\
& \left.-\frac{1}{2 \varepsilon} \int_{t-(L-x) / c}^{t} j_{y, \alpha}(s, x+c(t-s)) d s, \quad(t, x) \in \mathbb{R}_{t} \times\right] 0, L[ \\
B_{z}^{1}(t, x) & =\frac{1}{2 c}\left(h_{0}(t-x / c)-h_{L}(t-(L-x) / c)\right)
\end{aligned}
$$

$\mathrm{RR} \mathrm{n}^{\circ} 3554$ 


$$
\begin{aligned}
& -\frac{1}{2 c \varepsilon} \int_{t-x / c}^{t} j_{y, \alpha}(s, x-c(t-s)) d s \\
& \left.+\frac{1}{2 c \varepsilon} \int_{t-(L-x) / c}^{t} j_{y, \alpha}(s, x+c(t-s)) d s, \quad(t, x) \in \mathbb{R}_{t} \times\right] 0, L[
\end{aligned}
$$

and $j_{x, \alpha}=\int_{v} v_{x} f_{\alpha} d v, j_{y, \alpha}=\int_{v} v_{y} f_{\alpha} d v$ and $f_{\alpha}$ is the mild periodic solution for the following modified Vlasov problem:

$$
\begin{gathered}
\alpha f_{\alpha}+\partial_{t} f_{\alpha}+v_{x} \cdot \partial_{x} f_{\alpha}+\frac{q}{m}\left(R_{\alpha} E_{x}+v_{y} \cdot R_{\alpha} B_{z}\right) \cdot \partial_{v_{x}} f_{\alpha} \\
+\frac{q}{m}\left(R_{\alpha} E_{y}-v_{x} \cdot R_{\alpha} B_{z}\right) \cdot \partial_{v_{y}} f_{\alpha}=0, \\
\left.\left(t, x, v_{x}, v_{y}\right) \in \mathbb{R}_{t} \times\right] 0, L\left[\times \mathbb{R}_{v}^{2}\right. \\
f_{\alpha}\left(t, 0, v_{x}, v_{y}\right)=g_{0}\left(t, v_{x}, v_{y}\right), \quad t \in \mathbb{R}_{t} v_{x}>0, v_{y} \in \mathbb{R}_{v} \\
f_{\alpha}\left(t, L, v_{x}, v_{y}\right)=g_{L}\left(t, v_{x}, v_{y}\right), \quad t \in \mathbb{R}_{t} v_{x}<0, v_{y} \in \mathbb{R}_{v} .
\end{gathered}
$$

The term $\alpha \cdot f_{\alpha}$ changes the formulas (26) and (27) in the following way:

$$
\begin{aligned}
<f_{\alpha}, \varphi> & =\int_{0}^{T} d t \int_{v_{x}>0} \int_{v_{y}} d v \int_{t}^{\tau_{o, \alpha}} v_{x} \cdot g_{0}\left(t, v_{x}, v_{y}\right) e^{-\alpha(t-s)} \\
& \cdot \varphi\left(s, X_{\alpha}\left(s ; 0, v_{x}, v_{y}, t\right), V_{x, \alpha}\left(s ; 0, v_{x}, v_{y}, t\right), V_{y, \alpha}\left(s ; 0, v_{x}, v_{y}, t\right) d s\right. \\
& -\quad \int_{0}^{T} d t \int_{v_{x}<0} \int_{v_{y}} d v \int_{t}^{\tau_{o}, \alpha} v_{x} \cdot g_{L}\left(t, v_{x}, v_{y}\right) e^{-\alpha(t-s)} \\
& \cdot \varphi\left(s, X_{\alpha}\left(s ; L, v_{x}, v_{y}, t\right), V_{x, \alpha}\left(s ; L, v_{x}, v_{y}, t\right), V_{y, \alpha}\left(s ; L, v_{x}, v_{y}, t\right) d s\right.
\end{aligned}
$$




$$
f_{\alpha}\left(t, x, v_{x}, v_{y}\right)=\left\{\begin{aligned}
g_{0}\left(\tau_{i}^{\alpha}, V_{x, \alpha}\left(\tau_{i}^{\alpha} ; x,\right.\right. & \left.\left.v_{x}, v_{y}, t\right), V_{y, \alpha}\left(\tau_{i}^{\alpha} ; x, v_{x}, v_{y}, t\right)\right) e^{-\alpha\left(t-\tau_{i}^{\alpha}\right)} \\
& \text { if } \tau_{i}^{\alpha}>-\infty \text { and } X_{\alpha}\left(\tau_{i}^{\alpha} ; x, v_{x}, v_{y}, t\right)=0 \\
g_{L}\left(\tau_{i}^{\alpha}, V_{x, \alpha}\left(\tau_{i}^{\alpha} ; x,\right.\right. & \left.\left.v_{x}, v_{y}, t\right), V_{y, \alpha}\left(\tau_{i}^{\alpha} ; x, v_{x}, v_{y}, t\right)\right) e^{-\alpha\left(t-\tau_{i}^{\alpha}\right)} \\
& \text { if } \tau_{i}^{\alpha}>-\infty \text { and } X_{\alpha}\left(\tau_{i}^{\alpha} ; x, v_{x}, v_{y}, t\right)=L \\
0 . & \text { otherwise. }
\end{aligned}\right.
$$

Moreover, for the modified Vlasov problem, the law for the conservation of the total mass, obtained by multiplying and integrating over all $\left(v_{x}, v_{y}\right) \in \mathbb{R}_{v}^{2}$, produces:

$$
\left.\alpha \rho_{\alpha}+\partial_{t} \rho_{\alpha}+\partial_{x} j_{x, \alpha}=0,(t, x) \in \mathbb{R}_{t} \times\right] 0, L[
$$

or:

$$
\left.\partial_{t} \rho_{\alpha}+\partial_{x}\left(j_{x, \alpha}+\alpha \int \rho_{\alpha} d x\right)=0,(t, x) \in \mathbb{R}_{t} \times\right] 0, L[
$$

Now, if we want to preserve the divergence equation, it is clear that we have to add the extra term $\alpha \int \rho_{\alpha} d x$ in the definition of $E_{x}^{1}$ of (66). In order to assure the time periodicity for $\left(E_{x}^{1}, E_{y}^{1}, B_{z}^{1}\right)$, a supplementarily condition will be assumed.

Proposition 1 We assume that the following condition holds for $t \in \mathbb{R}_{t}$ :

$$
\int_{0}^{T} d t \int_{v_{x}>0} \int_{v_{y}} v_{x} g_{0}\left(t, v_{x}, v_{y}\right) d v+\int_{0}^{T} d t \int_{v_{x}<0} \int_{v_{y}} v_{x} g_{L}\left(t, v_{x}, v_{y}\right) d v=0
$$

Then, $\left(E_{x}^{1}, E_{y}^{1}, B_{z}^{1}\right)$ given by (66) are $T$ periodic and verify the Maxwell equations (55), (56) and the boundary conditions (59) and (60).

Proof Using Remark 3, we deduce that the mild solution of the modified Vlasov problem is $T$ periodic too. Now it is easy to check that $E_{y}^{1}$ and $B_{z}^{1}$ given by (66) are $T$ periodic and verify the Maxwell equations (55), (56) and the boundary conditions $(59),(60)$. In order to prove the periodicity of $E_{x}$,

$\mathrm{RR} \mathrm{n}^{\circ} 3554$ 
we use the continuity equation (72) for problem (67) whose solution is split in $f_{0}$ and $f_{L}$ as in Remark 5. By integration on $[0, T]$ we deduce:

$$
\partial_{x}\left(\int_{0}^{T}\left(\alpha \int_{0}^{x} \rho_{0}(t, y) d y+j_{x, 0}(t, x)\right) d t\right)=0
$$

and therefore:

$$
\begin{aligned}
\alpha \int_{0}^{T} d t \int_{0}^{x} \rho_{0}(t, y) d y+\int_{0}^{T} j_{x, 0}(t, x) d t & =\int_{0}^{T} j_{x, 0}(t, 0) d t \\
& =\int_{0}^{T} d t \int_{v_{x}>0} \int_{v_{y}} v_{x} g_{0}\left(t, v_{x}, v_{y}\right) d v
\end{aligned}
$$

where we have used (51). In the same way we obtain:

$$
\partial_{x}\left(\int_{0}^{T}\left(-\alpha \int_{x}^{L} \rho_{L}(t, y) d y+j_{x, L}(t, x)\right) d t\right)=0
$$

and:

$$
\begin{aligned}
-\alpha \int_{0}^{T} d t \int_{x}^{L} \rho_{L}(t, y) d y+\int_{0}^{T} j_{x, L}(t, x) d t & =\int_{0}^{T} j_{x, L}(t, L) d t \\
& =\int_{0}^{T} d t \int_{v_{x}<0} \int_{v_{y}} v_{x} g_{L}\left(t, v_{x}, v_{y}\right) d v
\end{aligned}
$$

Now, using (75), (77) and (73) we deduce:

$$
\int_{0}^{T}\left(j_{x, \alpha}(t, y)+\alpha \int_{0}^{x} \rho_{0}(t, y) d y-\alpha \int_{x}^{L} \rho_{L}(t, y) d y\right) d t=0
$$

and so $E_{x}^{1}$ given by $(66)$ is also $T$ periodic.

Remark 6 The electric field verifies the divergence equation:

$$
\left.\partial_{x} E_{x}^{1}=\frac{1}{\varepsilon} \rho(t, x), \quad(t, x) \in \mathbb{R}_{t} \times\right] 0, L[
$$


and the modified Maxwell equation:

$$
\begin{aligned}
\partial_{t} E_{x}=-\frac{1}{\varepsilon} j_{x, \alpha}-\frac{\alpha}{\varepsilon} \int_{0}^{x} \rho_{0, \alpha}(t, y) d y+\frac{\alpha}{\varepsilon} \int_{x}^{L} \rho_{L, \alpha}(t, y) d y, \\
\left.(t, x) \in \mathbb{R}_{t} \times\right] 0, L[
\end{aligned}
$$

Proof From (54) we have:

$$
\begin{aligned}
\partial_{x} E_{x}^{1} & =-\frac{1}{\varepsilon} \int_{0}^{t}\left(\partial_{x} j_{x, \alpha}(s, x)+\alpha \rho_{0}(s, x)+\alpha \rho_{L}(s, x)\right) d s+\frac{1}{\varepsilon} \rho(0, x) \\
& =-\frac{1}{\varepsilon} \int_{0}^{t}\left(\partial_{x} j_{x, \alpha}(s, x)+\alpha \rho_{\alpha}(s, x)\right) d s+\frac{1}{\varepsilon} \rho(0, x) \\
& =\frac{1}{\varepsilon} \int_{0}^{t} \partial_{t} \rho_{\alpha}(s, x) d s+\frac{1}{\varepsilon} \rho(0, x) \\
& =\frac{1}{\varepsilon} \rho(t, x) .
\end{aligned}
$$

The second formula can be easily checked using (54). We prove that the application $F$ maps $\mathcal{X}$ into itself and is continuous in $L^{2}\left(\mathbb{R}_{t} \times\right] 0, L[)$ in respect with the weak topology.

Lemma 2 We assume (30), (73), that the constant $K$ which defines the set $\mathcal{X}$ verifies:

$$
K+\frac{K}{c} \cdot\left(v_{1}+\frac{q}{m} \cdot K \cdot \frac{2 L}{v_{0}}\right) \leq \frac{m v_{0}^{2}}{4 q L}
$$

Then if $g_{0}, g_{L}, h_{0}, h_{L}$ satisfy

$\frac{1}{\varepsilon} \cdot \frac{\pi}{2} \cdot q\left(\left\|g_{0}\right\|_{L^{\infty}}+\left\|g_{L}\right\|_{L^{\infty}}\right) \cdot\left(v_{1}+v_{0} / 2\right)^{2}\left(T\left(v_{1}+v_{0} / 2\right)+\alpha L T+L\right) \leq \frac{K}{\sqrt{2}}$

$\frac{1}{2 \varepsilon} \cdot \frac{L}{c} \cdot \frac{\pi}{2} \cdot q\left(\left\|g_{0}\right\|_{L^{\infty}}+\left\|g_{L}\right\|_{L^{\infty}}\right) \cdot\left(v_{1}+v_{0} / 2\right)^{3}+\frac{1}{2}\left(\left\|h_{0}\right\|_{L^{\infty}}+\left\|h_{L}\right\|_{L^{\infty}}\right) \leq \frac{K}{\sqrt{2}}$

the set $\mathcal{X}$ is invariant by the application $F(F(\mathcal{X}) \subset \mathcal{X})$.

$\mathrm{RR} \mathrm{n}^{\circ} 3554$ 
Proof From Corollary 2 applied to the regularized field (63) we obtain the following estimates:

$$
\begin{aligned}
\left\|E_{x}^{1}\right\|_{L^{\infty}} \leq & \frac{1}{\varepsilon} \cdot \frac{\pi}{2} \cdot q\left(\left\|g_{0}\right\|_{L^{\infty}}+\left\|g_{L}\right\|_{L^{\infty}}\right) \cdot\left(v_{1}+v_{0} / 2\right)^{2} \\
& \left(T\left(v_{1}+v_{0} / 2\right)+\alpha L T+L\right) \leq \frac{K}{\sqrt{2}} \\
\left\|E_{y}^{1}\right\|_{L^{\infty}} \leq & \frac{1}{2 \varepsilon} \cdot \frac{L}{c} \cdot \frac{\pi}{2} \cdot q\left(\left\|g_{0}\right\|_{L^{\infty}}+\left\|g_{L}\right\|_{L^{\infty}}\right) \cdot\left(v_{1}+v_{0} / 2\right)^{3} \\
+ & \frac{1}{2}\left(\left\|h_{0}\right\|_{L^{\infty}}+\left\|h_{L}\right\|_{L^{\infty}}\right) \leq \frac{K}{\sqrt{2}}
\end{aligned}
$$

Therefore, we have:

$$
\begin{aligned}
\left\|E^{1}\right\|_{L^{\infty}}=\left\|\sqrt{\left|E_{x}^{1}\right|^{2}+\left|E_{y}^{1}\right|^{2}}\right\|_{L^{\infty}} \leq K \\
c \cdot\left\|B_{z}^{1}\right\|_{L^{\infty}} \leq \frac{1}{2 \varepsilon} \cdot \frac{L}{c} \cdot \frac{\pi}{2} \cdot q\left(\left\|g_{0}\right\|_{L^{\infty}}+\left\|g_{L}\right\|_{L^{\infty}}\right) \cdot\left(v_{1}+v_{0} / 2\right)^{3} \\
+\frac{1}{2}\left(\left\|h_{0}\right\|_{L^{\infty}}+\left\|h_{L}\right\|_{L^{\infty}}\right) \leq K
\end{aligned}
$$

Moreover, using Proposition 1 we deduce that $F\left(E_{x}, E_{y}, B_{z}\right)$ is also $T$ periodic, so $F(\mathcal{X}) \subset \mathcal{X}$.

For the proof of the continuity we need the following Lemma concerning the uniqueness of weak solution for the modified Vlasov equation:

Lemma 3 Let $\left(E_{x}, E_{y}, B_{z}\right) \in\left(L^{\infty}\left(\mathbb{R}_{t} ; W^{1, \infty}(] 0, L[)\right)\right)^{3}$ and $g_{0}, g_{L} \in L^{\infty}\left(\mathbb{R}_{t} \times\right.$ $\left.\Sigma^{-}\right)$be $T$ periodic functions which verify (28), (30). Then a weak periodic solution in $L^{\infty}\left(\mathbb{R}_{t} \times\right] 0, L\left[\times \mathbb{R}_{v}^{2}\right)$ of the modified Vlasov equation (67) is unique and therefore is the mild solution given by (70).

Proof Assume that $f_{\alpha}$ is a solution in $L^{\infty}\left(\mathbb{R}_{t} \times\right] 0, L\left[\times \mathbb{R}_{v}^{2}\right)$ with $g_{0}=0$ and $g_{L}=0$. We have :

$$
\begin{aligned}
\partial_{t} f_{\alpha} & +v_{x} \cdot \partial_{x} f_{\alpha}+\frac{q}{m}\left(R_{\alpha} E_{x}+v_{y} \cdot R_{\alpha} B_{z}\right) \cdot \partial_{v_{x}} f_{\alpha} \\
& +\frac{q}{m}\left(R_{\alpha} E_{y}-v_{x} \cdot R_{\alpha} B_{z}\right) \cdot \partial_{v_{y}} f_{\alpha}=-\alpha f_{\alpha} \in L^{\infty}\left(\mathbb{R}_{t} \times\right] 0, L\left[\times \mathbb{R}_{v}^{2}\right),
\end{aligned}
$$


and therefore( cf. [2], [3]) we obtain:

$$
\begin{aligned}
-\alpha \cdot f_{\alpha}^{2}=f_{\alpha}\left(\partial_{t} f_{\alpha}\right. & +v_{x} \cdot \partial_{x} f_{\alpha}+\frac{q}{m}\left(R_{\alpha} E_{x}+v_{y} \cdot R_{\alpha} B_{z}\right) \cdot \partial_{v_{x}} f_{\alpha} \\
& \left.+\frac{q}{m}\left(R_{\alpha} E_{y}-v_{x} \cdot R_{\alpha} B_{z}\right) \cdot \partial_{v_{y}} f_{\alpha}\right) \\
& =\frac{1}{2}\left(\partial_{t} f_{\alpha}^{2}+v_{x} \cdot \partial_{x} f_{\alpha}^{2}+\frac{q}{m}\left(R_{\alpha} E_{x}+v_{y} \cdot R_{\alpha} B_{z}\right) \cdot \partial_{v_{x}} f_{\alpha}^{2}\right. \\
& \left.+\frac{q}{m}\left(R_{\alpha} E_{y}-v_{x} \cdot R_{\alpha} B_{z}\right) \cdot \partial_{v_{y}} f_{\alpha}^{2}\right) .
\end{aligned}
$$

Integrating this relation on $] 0, T[\times] 0, L\left[\times \mathbb{R}_{v}^{2}\right.$ gives:

$$
\begin{aligned}
\alpha \int_{0}^{T} \int_{0}^{L} \int_{\mathbb{R}_{v}^{2}} f_{\alpha}^{2} d v d x d t= & -\frac{1}{2} \int_{0}^{T} \int_{v_{x}>0} \int_{v_{y}} v_{x} f_{\alpha}^{2}\left(t, L, v_{x}, v_{y}\right) d v d t \\
& +\frac{1}{2} \int_{0}^{T} \int_{v_{x}<0} \int_{v_{y}} v_{x} f_{\alpha}^{2}\left(t, 0, v_{x}, v_{y}\right) d v d t \leq 0 .
\end{aligned}
$$

Now we can prove the continuity of the application $F$. We have the following proposition:

Proposition 2 Let $g_{0}, g_{L}, h_{0}, h_{L} \in L^{\infty}\left(\mathbb{R}_{t} \times \Sigma^{-}\right)$be $T$ periodic functions and $v_{0}, v_{1}, K$ constants which verify (30), (81) and (73). Then the application $F$ is continuous with respect to the weak topology of $L_{T}^{2}\left(\mathbb{R}_{t} \times\right] 0, L[)^{3}$.

Proof. Let $\left(E_{x}^{n}, E_{y}^{n}, B_{z}^{n}\right)_{n \geq 1} \subset \mathcal{X}$ such as:

$$
\left(E_{x}^{n}, E_{y}^{n}, B_{z}^{n}\right) \rightarrow\left(E_{x}, E_{y}, B_{z}\right), \text { weak in }\left(L_{T}^{2}\right)^{3}
$$

For the regularized field we have the pointwise convergence :

$\left(R_{\alpha} E_{x}^{n}, R_{\alpha} E_{y}^{n}, R_{\alpha} B_{z}^{n}\right)(t, x) \rightarrow\left(R_{\alpha} E_{x}, R_{\alpha} E_{y}, R_{\alpha} B_{z}\right)(t, x), \quad \forall(t, x) \in[0, T] \times[0, L]$,

and therefore, by the dominate convergence theorem we obtain:

$$
\left(R_{\alpha} E_{x}^{n}, R_{\alpha} E_{y}^{n}, R_{\alpha} B_{z}^{n}\right) \rightarrow\left(R_{\alpha} E_{x}, R_{\alpha} E_{y}, R_{\alpha} B_{z}\right), \text { strong } \operatorname{in}\left(L_{T}^{2}\right)^{3}
$$

Denote by $f^{n}, f$ the mild solution given by (70) associated to the field $\left(R_{\alpha} E_{x}^{n}\right.$, $\left.R_{\alpha} E_{y}^{n}, R_{\alpha} B_{z}^{n}\right)$ and $\left(R_{\alpha} E_{x}, R_{\alpha} E_{y}, R_{\alpha} B_{z}\right)$. We recall that $g_{0}, g_{L}$ are bounded 
in $L^{\infty}$, and therefore, $\left(f^{n}\right)_{n \geq 1}$ is uniformly bounded in $L^{\infty}\left(\mathbb{R}_{t} \times\right] 0, L\left[\times \mathbb{R}_{v^{2}}\right)$. After extracting a subsequence if necessary, we have:

$$
f^{n} \rightarrow \tilde{f}, \text { weak } \star \text { in } L^{\infty} .
$$

Moreover, because $f^{n}$ have uniformly bounded support in $v$, we deduce that:

$$
\rho^{n}:=q \int_{\mathbb{R}_{v^{2}}} f^{n} d v \rightarrow \tilde{\rho}:=q \int_{\mathbb{R}_{v^{2}}} \tilde{f} d v \text { weak } \star \text { in } L^{\infty},
$$

and:

$$
j_{x, y}^{n}:=q \int_{\mathbb{R}_{v^{2}}} v_{x, y} f^{n} d v \rightarrow \tilde{j}_{x, y}:=q \int_{\mathbb{R}_{v^{2}}} v_{x, y} \tilde{f} d v \text { weak } \star \text { in } L^{\infty} .
$$

Now we can prove that $\tilde{f}$ is the mild solution of the modified Vlasov problem corresponding to the field $\left(R_{\alpha} E_{x}, R_{\alpha} E_{y}, R_{\alpha} B_{z}\right)$. Because $f^{n}$ is the mild solution, it is also a weak solution:

$$
\begin{aligned}
\int_{0}^{T} \int_{0}^{L} \int_{\mathbb{R}_{v}^{2}}\left(-\alpha \cdot \theta+\partial_{t} \theta+v_{x} \cdot \partial_{x} \theta\right. & +\frac{q}{m}\left(R_{\alpha} E_{x}^{n}+v_{y} \cdot R_{\alpha} B_{z}^{n}\right) \cdot \partial_{v_{x}} \theta \\
& \left.+\frac{q}{m}\left(R_{\alpha} E_{y}^{n}-v_{x} \cdot R_{\alpha} B_{z}^{n}\right) \cdot \partial_{v_{y}} \theta\right) f^{n} d v d x d t \\
& =\int_{0}^{T} \int_{v_{x}<0} \int_{v_{y}} v_{x} \cdot g_{L} \theta\left(t, L, v_{x}, v_{y}\right) d v d t \\
& -\int_{0}^{T} \int_{v_{x}>0} \int_{v_{y}} v_{x} \cdot g_{0} \theta\left(t, 0, v_{x}, v_{y}\right) d v d t
\end{aligned}
$$

for all $T$ periodic function $\theta \in \mathcal{V}$. We have:

$$
\begin{aligned}
\lim _{n \rightarrow \infty} & \int_{0}^{T} \int_{0}^{L} \int_{\mathbb{R}_{v}^{2}} f^{n} \cdot\left(-\alpha \cdot \theta+\partial_{t} \theta+v_{x} \cdot \partial_{x} \theta\right) d v d x d t \\
= & \int_{0}^{T} \int_{0}^{L} \int_{\mathbb{R}_{v}^{2}} \tilde{f} \cdot\left(-\alpha \cdot \theta+\partial_{t} \theta+v_{x} \cdot \partial_{x} \theta\right) d v d x d t
\end{aligned}
$$

For the other terms we remark that $\int_{\mathbb{R}_{v}^{2}} \partial_{v_{x}} \theta \cdot f^{n} d v$ and $\int_{\mathbb{R}_{v}^{2}} \partial_{v_{y}} \theta \cdot f^{n} d v$ converge in $L_{T}^{2}$ weak. Therefore using (85) we get:

$$
\lim _{n \rightarrow \infty} \int_{0}^{T} \int_{0}^{L} \int_{\mathbb{R}_{v}^{2}} \frac{q}{m}\left(R_{\alpha} E_{x}^{n}+v_{y} \cdot R_{\alpha} B_{z}^{n}\right) \partial_{v_{x}} \theta \cdot f^{n} d v d x d t
$$




$$
\begin{aligned}
& =\lim _{n \rightarrow \infty}<\frac{q}{m} R_{\alpha} E_{x}^{n}, \int_{\mathbb{R}_{v}^{2}} \partial_{v_{x}} \theta \cdot f^{n} d v>_{L_{T}^{2}} \\
& +\lim _{n \rightarrow \infty}<\frac{q}{m} R_{\alpha} B_{z}^{n}, \int_{\mathbb{R}_{v}^{2}} v_{y} \cdot \partial_{v_{x}} \theta \cdot f^{n} d v>_{L_{T}^{2}} \\
& =<\frac{q}{m} R_{\alpha} E_{x}, \int_{\mathbb{R}_{v}^{2}} \partial_{v_{x}} \theta \cdot \tilde{f} d v>_{L_{T}^{2}} \\
& +\quad<\frac{q}{m} R_{\alpha} B_{z}, \int_{\mathbb{R}_{v}^{2}} v_{y} \cdot \partial_{v_{x}} \theta \cdot \tilde{f} d v>_{L_{T}^{2}} \\
& =\int_{0}^{T} \int_{0}^{L} \int_{\mathbb{R}_{v}^{2}} \frac{q}{m}\left(R_{\alpha} E_{x}+v_{y} \cdot R_{\alpha} B_{z}\right) \partial_{v_{x}} \theta \cdot \tilde{f} d v d x d t
\end{aligned}
$$

Therefore, $\tilde{f}$ is a weak solution for the modified Vlasov problem corresponding to the field $\left(R_{\alpha} E_{x}, R_{\alpha} E_{y}, R_{\alpha} B_{z}\right)$ :

$$
\begin{aligned}
\int_{0}^{T} \int_{0}^{L} \int_{\mathbb{R}_{v}^{2}}\left(-\alpha \cdot \theta+\partial_{t} \theta+v_{x} \cdot \partial_{x} \theta\right. & +\frac{q}{m}\left(R_{\alpha} E_{x}+v_{y} \cdot R_{\alpha} B_{z}\right) \cdot \partial_{v_{x}} \theta \\
& \left.+\frac{q}{m}\left(R_{\alpha} E_{y}-v_{x} \cdot R_{\alpha} B_{z}\right) \cdot \partial_{v_{y}} \theta\right) \tilde{f} d v d x d t \\
& =\int_{0}^{T} \int_{v_{x}<0} \int_{v_{y}} v_{x} \cdot g_{L} \theta\left(t, L, v_{x}, v_{y}\right) d v d t \\
& -\int_{0}^{T} \int_{v_{x}>0} \int_{v_{y}} v_{x} \cdot g_{0} \theta\left(t, 0, v_{x}, v_{y}\right) d v d t
\end{aligned}
$$

for all $T$ periodic function $\theta \in \mathcal{V}$. But using Lemma 3 we deduce that $\tilde{f}$ is the mild solution corresponding to the field $\left(R_{\alpha} E_{x}, R_{\alpha} E_{y}, R_{\alpha} B_{z}\right)$ ( uniqueness of the weak solution for the modified Vlasov problem ), so $\tilde{f}=f$ and we have:

$$
j_{x, y}^{n}:=q \int_{\mathbb{R}_{v^{2}}} v_{x, y} f^{n} d v \rightarrow j_{x, y}:=q \int_{\mathbb{R}_{v^{2}}} v_{x, y} f d v \quad \text { weak } \star \text { in } L^{\infty} .
$$

Now, it is easy to check that we can pass to the limit in (66) to obtain:

$$
\lim _{n \rightarrow \infty} F\left(E_{x}^{n}, E_{y}^{n}, B_{z}^{n}\right)=F\left(E_{x}, E_{y}, B_{z}\right) \text {, weak in } L_{T}^{2} .
$$

Proposition 3 Let $g_{0}, g_{L}, h_{0}, h_{L} \in L^{\infty}\left(\mathbb{R}_{t} \times \Sigma^{-}\right)$be $T$ periodic functions and $v_{0}, v_{1}, K$ constants which verify (30), (81), (82), (83) and (73). Then the modified $1 D$ Vlasov-Maxwell system has at least one weak periodic solution.

$\mathrm{RR} \mathrm{n}^{\circ} 3554$ 
Proof. It is an immediate consequence of Schauder fixed point theorem.

\section{Weak periodic solutions for the classical 1D Vlasov-Maxwell system.}

We prove the existence of periodic weak solution for the Vlasov-Maxwell system. Obviously, this result is a direct consequence of Proposition 3.

Theorem 1 Let $g_{0}, g_{L}, h_{0}, h_{L} \in L^{\infty}\left(\mathbb{R}_{t} \times \Sigma^{-}\right)$be $T$ periodic functions and $v_{0}, v_{1}, K$ constants which verify:

$$
\begin{gathered}
\operatorname{supp}\left(g_{0}\right) \subset\left\{\left(t, x, v_{x}, v_{y}\right) ; t \in \mathbb{R}_{t}, x=0,0<v_{0} \leq v_{x}, \sqrt{v_{x}^{2}+v_{y}^{2}} \leq v_{1}\right\} \\
\operatorname{supp}\left(g_{L}\right) \subset\left\{\left(t, x, v_{x}, v_{y}\right) ; t \in \mathbb{R}_{t}, x=L, 0>-v_{0} \geq v_{x}, \sqrt{v_{x}^{2}+v_{y}^{2}} \leq v_{1}\right\}, \\
K+\frac{K}{c} \cdot\left(v_{1}+\frac{q}{m} \cdot K \cdot \frac{2 L}{v_{0}}\right) \leq \frac{m v_{0}^{2}}{4 q L}, \\
\frac{1}{\varepsilon} \cdot \frac{\pi}{2} \cdot q\left(\left\|g_{0}\right\|_{L^{\infty}}+\left\|g_{L}\right\|_{L^{\infty}}\right) \cdot\left(v_{1}+v_{0} / 2\right)^{2}\left(T\left(v_{1}+v_{0} / 2\right)+L\right)<\frac{K}{\sqrt{2}}, \\
\frac{1}{2 \varepsilon} \cdot \frac{L}{c} \cdot \frac{\pi}{2} \cdot q\left(\left\|g_{0}\right\|_{L^{\infty}}+\left\|g_{L}\right\|_{L^{\infty}}\right) \cdot\left(v_{1}+v_{0} / 2\right)^{3}+\frac{1}{2}\left(\left\|h_{0}\right\|_{L^{\infty}}+\left\|h_{L}\right\|_{L^{\infty}}\right) \leq \frac{K}{\sqrt{2}}
\end{gathered}
$$

and:

$$
\int_{0}^{T} d t \int_{v_{x}>0} \int_{v_{y}} v_{x} g_{0}\left(t, v_{x}, v_{y}\right) d v+\int_{0}^{T} d t \int_{v_{x}<0} \int_{v_{y}} v_{x} g_{L}\left(t, v_{x}, v_{y}\right) d v=0 .
$$

Then the classical 1D Vlasov-Maxwell system has at least one weak periodic solution. 
Proof. Let $\left(\alpha_{n}\right)_{n \geq 1}$ be a sequence of positive numbers, whose limit is 0 . We observe that for $\alpha_{n}$ small we have:

$\frac{1}{\varepsilon} \cdot \frac{\pi}{2} \cdot q\left(\left\|g_{0}\right\|_{L^{\infty}}+\left\|g_{L}\right\|_{L^{\infty}}\right) \cdot\left(v_{1}+v_{0} / 2\right)^{2}\left(T\left(v_{1}+v_{0} / 2\right)+\alpha_{n} L T+L\right) \leq \frac{K}{\sqrt{2}}$

Therefore, by Proposition 3, there is $\left(f^{n}, E_{x}^{n}, E_{y}^{n}, B_{z}^{n}\right)$ weak periodic solutions for the $\alpha_{n}$ regularized Vlasov-Maxwell system:

$$
\begin{gathered}
\alpha_{n} \cdot f^{n}+\partial_{t} f^{n}+v_{x} \cdot \partial_{x} f^{n}+\frac{q}{m}\left(R_{\alpha_{n}} E_{x}^{n}+v_{y} \cdot R_{\alpha_{n}} B_{z}^{n}\right) \cdot \partial_{v_{x}} f^{n} \\
+\frac{q}{m}\left(R_{\alpha_{n}} E_{y}^{n}-v_{x} \cdot R_{\alpha_{n}} B_{z}^{n}\right) \cdot \partial_{v_{y}} f^{n}=0, \\
\left.\left(t, x, v_{x}, v_{y}\right) \in \mathbb{R}_{t} \times\right] 0, L\left[\times \mathbb{R}_{v}^{2},\right. \\
\partial_{t} E_{x}^{n}=-\frac{1}{\varepsilon} j_{x}^{n}-\frac{\alpha_{n}}{\varepsilon} \int_{0}^{x} \rho_{0}^{n}(t, y) d y+\frac{\alpha_{n}}{\varepsilon} \int_{x}^{L} \rho_{L}^{n}(t, y) d y, \\
\left.\partial_{t} E_{y}^{n}+c^{2} \partial_{x} B_{z}^{n}=-\frac{1}{\varepsilon} j_{y}^{n}:=-\frac{1}{\varepsilon} \int_{v} v_{y} f^{n}\left(t, x, v_{x}, v_{y}\right) d v, \quad(t, x) \in \mathbb{R}_{t} \times\right] 0, L[, \\
\left.\partial_{t} B_{z}^{n}+\partial_{x} E_{y}^{n}=0, \quad(t, x) \in \mathbb{R}_{t} \times\right] 0, L[,
\end{gathered}
$$

with the boundary conditions:

$$
\begin{gathered}
f^{n}\left(t, 0, v_{x}, v_{y}\right)=g_{0}\left(t, v_{x}, v_{y}\right), \quad t \in \mathbb{R}_{t} v_{x}>0, v_{y} \in \mathbb{R}_{v} \\
f^{n}\left(t, L, v_{x}, v_{y}\right)=g_{L}\left(t, v_{x}, v_{y}\right), \quad t \in \mathbb{R}_{t} v_{x}<0, v_{y} \in \mathbb{R}_{v} \\
E_{y}^{n}(t, 0)+c B_{z}^{n}(t, 0)=h_{0}(t), \quad t \in \mathbb{R}_{t}
\end{gathered}
$$

$\mathrm{RR} \mathrm{n}^{\circ} 3554$ 


$$
E_{y}^{n}(t, L)-c B_{z}^{n}(t, L)=h_{L}(t), \quad t \in \mathbb{R}_{t},
$$

After extracting subsequence, we have the convergence:

$$
\left(E_{x}^{n}, E_{y}^{n}, B_{z}^{n}\right) \rightarrow\left(E_{x}, E_{y}, B_{z}\right), \text { weak in }\left(L_{T}^{2}\right)^{3}
$$

and:

$$
f^{n} \rightarrow f, \text { weak } \star \text { in } L^{\infty} \text {. }
$$

Moreover, by regularization with $\alpha_{n} \rightarrow 0$, the first convergences are preserved:

$$
\begin{aligned}
\left|<R_{\alpha_{n}} E_{x}^{n}, \eta>_{L_{T}^{2}}-<E_{x}, \eta>_{L_{T}^{2}}\right| & =\left|<E_{x}^{n}, R_{\alpha_{n}} \eta>-<E_{x}, \eta>\right| \\
& =\left|<E_{x}^{n}, R_{\alpha_{n}} \eta-\eta>+<E_{x}^{n}-E_{x}, \eta>\right| \\
& \leq\left|<E_{x}^{n}-E_{x}, \eta>\right| \\
& +\left\|E_{x}^{n}\right\| \cdot\left\|R_{\alpha_{n}} \eta-\eta\right\| \rightarrow 0,
\end{aligned}
$$

and so:

$$
\left(R_{\alpha_{n}} E_{x}^{n}, R_{\alpha_{n}} E_{y}^{n}, R_{\alpha_{n}} B_{z}^{n}\right) \rightarrow\left(E_{x}, E_{y}, B_{z}\right) \text {, weak in }\left(L_{T}^{2}\right)^{3} .
$$

Because $f^{n}$ have uniformly bounded support in $v$, we deduce that:

$$
\rho^{n}:=q \int_{\mathbb{R}_{v^{2}}} f^{n} d v \rightarrow \rho:=q \int_{\mathbb{R}_{v^{2}}} f d v \text { weak } \star \text { in } L^{\infty}
$$

and:

$$
j_{x, y}^{n}:=q \int_{\mathbb{R}_{v^{2}}} v_{x, y} f^{n} d v \rightarrow j_{x, y}:=q \int_{\mathbb{R}_{v^{2}}} v_{x, y} f d v \text { weak } \star \text { in } L^{\infty} .
$$

The velocity average lemma of DiPerna and Lions [14] allows us to write:

$$
\rho^{n}:=q \int_{\mathbb{R}_{v^{2}}} f^{n} d v \rightarrow \rho:=q \int_{\mathbb{R}_{v^{2}}} f d v \text { in } L_{T}^{2},
$$

and:

$$
j_{x, y}^{n}:=q \int_{\mathbb{R}_{v^{2}}} v_{x, y} f^{n} d v \rightarrow j_{x, y}:=q \int_{\mathbb{R}_{v^{2}}} v_{x, y} f d v \text { in } L_{T}^{2}
$$


Moreover, we have:

$$
\int_{\mathbb{R}_{v^{2}}} \psi\left(v_{x}, v_{y}\right) f^{n} d v \rightarrow \int_{\mathbb{R}_{v^{2}}} \psi\left(v_{x}, v_{y}\right) f d v \text { in } L_{T}^{2}
$$

for all continuous function $\psi \in C\left(\mathbb{R}_{v^{2}}\right)$. We prove now that $f$ is a weak solution for the Vlasov problem corresponding to the field $\left(E_{x}, E_{y}, B_{z}\right)$. By a simple density argument, it is sufficient to consider test functions with a product structure (see [14]) :

$$
\theta\left(t, x, v_{x}, v_{y}\right)=\varphi(t, x) \cdot \psi\left(v_{x}, v_{y}\right)
$$

We have :

$$
\begin{aligned}
\lim _{n \rightarrow \infty} \int_{0}^{T} \int_{0}^{L} \int_{\mathbb{R}_{v}^{2}} & \frac{q}{m}\left(R_{\alpha} E_{x}^{n}+v_{y} \cdot R_{\alpha} B_{z}^{n}\right) \partial_{v_{x}} \theta \cdot f^{n} d v d x d t \\
= & \lim _{n \rightarrow \infty}<\frac{q}{m} R_{\alpha} E_{x}^{n}, \varphi(t, x) \int_{\mathbb{R}_{v}^{2}} \partial_{v_{x}} \psi \cdot f^{n} d v>_{L_{T}^{2}} \\
+ & \lim _{n \rightarrow \infty}<\frac{q}{m} R_{\alpha} B_{z}^{n}, \varphi(t, x) \int_{\mathbb{R}_{v}^{2}} v_{y} \cdot \partial_{v_{x}} \psi \cdot f^{n} d v>_{L_{T}^{2}} \\
= & <\frac{q}{m} R_{\alpha} E_{x}, \varphi(t, x) \int_{\mathbb{R}_{v}^{2}} \partial_{v_{x}} \psi \cdot f d v>_{L_{T}^{2}} \\
& +<\frac{q}{m} R_{\alpha} B_{z}, \varphi(t, x) \int_{\mathbb{R}_{v}^{2}} v_{y} \cdot \partial_{v_{x}} \psi \cdot f d v>_{L_{T}^{2}} \\
= & \int_{0}^{T} \int_{0}^{L} \int_{\mathbb{R}_{v}^{2}} \frac{q}{m}\left(R_{\alpha} E_{x}+v_{y} \cdot R_{\alpha} B_{z}\right) \partial_{v_{x}} \theta \cdot f d v d x d t
\end{aligned}
$$

In addition we have :

$$
\lim _{n \rightarrow \infty} \alpha_{n} \int_{0}^{T} \int_{0}^{L} \int_{\mathbb{R}_{v}^{2}} f^{n} \cdot \theta d v d x d t=0 .
$$

for all $T$ periodic function $\theta \in \mathcal{V}$. Furthermore, passing to the limit for $n \rightarrow \infty$ in (66) and using (94) and (95), we deduce the following equalities in $L_{T}^{2}$ :

$$
\begin{aligned}
E_{x}(t, x)= & -\frac{1}{\varepsilon} \int_{0}^{t} j_{x}(s, x) d s \\
& \left.+\frac{1}{\varepsilon} \int_{0}^{x} \rho(0, y) d y, \quad(t, x) \in \mathbb{R}_{t} \times\right] 0, L[
\end{aligned}
$$

$\mathrm{RR} \mathrm{n}^{\circ} 3554$ 


$$
\begin{aligned}
E_{y}(t, x) & =\frac{1}{2}\left(h_{0}(t-x / c)+h_{L}(t-(L-x) / c)\right) \\
& -\frac{1}{2 \varepsilon} \int_{t-x / c}^{t} j_{y}(s, x-c(t-s)) d s \\
& \left.-\frac{1}{2 \varepsilon} \int_{t-(L-x) / c}^{t} j_{y}(s, x+c(t-s)) d s, \quad(t, x) \in \mathbb{R}_{t} \times\right] 0, L[ \\
B_{z}(t, x) & =\frac{1}{2 c}\left(h_{0}(t-x / c)-h_{L}(t-(L-x) / c)\right) \\
& -\frac{1}{2 c \varepsilon} \int_{t-x / c}^{t} j_{y}(s, x-c(t-s)) d s \\
& \left.+\frac{1}{2 c \varepsilon} \int_{t-(L-x) / c}^{t} j_{y}(s, x+c(t-s)) d s, \quad(t, x) \in \mathbb{R}_{t} \times\right] 0, L[
\end{aligned}
$$

and so the field $\left(E_{x}, E_{y}, B_{z}\right)$ verifies the Maxwell equations.

\section{Weak periodic solutions for the relativistic 1D Vlasov-Maxwell system.}

Our arguments apply also to the relativistic $1 D$ Vlasov-Maxwell system:

$$
\begin{array}{r}
\partial_{t} f+V_{x}(p) \cdot \partial_{x} f+q\left(E_{x}+V_{y}(p) \cdot B_{z}\right) \cdot \partial_{p_{x}} f+q\left(E_{y}-V_{x}(p) \cdot B_{z}\right) \cdot \partial_{p_{y}} f=0, \\
\left.\left(t, x, p_{x}, p_{y}\right) \in \mathbb{R}_{t} \times\right] 0, L\left[\times \mathbb{R}_{p}^{2},\right.
\end{array}
$$


with the boundary conditions:

$$
\begin{gathered}
f\left(t, 0, p_{x}, p_{y}\right)=g_{0}\left(t, p_{x}, p_{y}\right), \quad t \in \mathbb{R}_{t} p_{x}>0, p_{y} \in \mathbb{R}_{p}, \\
f\left(t, L, p_{x}, p_{y}\right)=g_{L}\left(t, p_{x}, p_{y}\right), \quad t \in \mathbb{R}_{t} p_{x}<0, p_{y} \in \mathbb{R}_{p}, \\
E_{y}(t, 0)+c B_{z}(t, 0)=h_{0}(t), \quad t \in \mathbb{R}_{t}, \\
E_{y}(t, L)-c B_{z}(t, L)=h_{L}(t), \quad t \in \mathbb{R}_{t},
\end{gathered}
$$

where $g_{0}, g_{L}, h_{0}, h_{L}$ are $T$ periodic functions and the velocity $V(p)$ is given by:

$$
V(p)=\left(V_{x}(p), V_{y}(p)\right)=c \cdot \frac{\left(p_{x}, p_{y}\right)}{\sqrt{m^{2} c^{2}+\|p\|^{2}}}, \quad\left(p_{x}, p_{y}\right) \in \mathbb{R}_{p^{2}}
$$

We only have to modify the preceeding proofs slightly. First, we observe that the quadratic nonlinear term $(E(t, x)+V(p) \wedge B(t, x)) \cdot \nabla_{p} f$ may be recast as an exact derivation:

$$
(E(t, x)+V(p) \wedge B(t, x)) \cdot \nabla_{p} f=\nabla_{p} \cdot\{(E(t, x)+V(p) \wedge B(t, x)) \cdot f\} .
$$

Definition 3 Let $E_{x}, E_{y}, B_{z} \in L^{\infty}\left(\mathbb{R}_{t} \times\right] 0, L[)$ and $g_{0}, g_{L} \in L_{l o c}^{1}\left(\mathbb{R}_{t} \times \Sigma^{-}\right)$be $T$ periodic functions in time, where:

$$
\begin{aligned}
\Sigma^{-} & =\left\{\left(t, x, p_{x}, p_{y}\right) \mid t \in \mathbb{R}, x=0, p_{x}>0, p_{y} \in \mathbb{R}\right\} \\
& \cup\left\{\left(t, x, p_{x}, p_{y}\right) \mid t \in \mathbb{R}, x=L, p_{x}<0, p_{y} \in \mathbb{R}\right\} .
\end{aligned}
$$

We say that $f \in L_{\text {loc }}^{1}\left(\mathbb{R}_{t} \times\right] 0, L\left[\times \mathbb{R}_{p}^{2}\right)$ is a $T$ periodic weak solution of problem (98), (102), (103) iff:

$\mathrm{RR} \mathrm{n}^{\circ} 3554$ 


$$
\begin{aligned}
\int_{0}^{T} \int_{0}^{L} \int_{\mathbb{R}_{p}^{2}}\left(\partial_{t} \theta+V_{x}(p) \cdot \partial_{x} \theta\right. & +q\left(E_{x}+V_{y}(p) \cdot B_{z}\right) \cdot \partial_{p_{x}} \theta \\
& \left.+q\left(E_{y}-V_{x}(p) \cdot B_{z}\right) \cdot \partial_{p_{y}} \theta\right) f\left(t, x, p_{x}, p_{y}\right) d p d x d t \\
& =\int_{0}^{T} \int_{p_{x}<0} \int_{p_{y}} V_{x}(p) g_{L}\left(t, p_{x}, p_{y}\right) \theta\left(t, L, p_{x}, p_{y}\right) d p d t \\
& -\int_{0}^{T} \int_{p_{x}>0} \int_{p_{y}} V_{x}(p) g_{0}\left(t, p_{x}, p_{y}\right) \theta\left(t, 0, p_{x}, p_{y}\right) d p d t
\end{aligned}
$$

for all $T$ periodic function $\theta \in \mathcal{V}$, where:

$$
\begin{array}{r}
\mathcal{V}=\left\{\eta \in W^{1, \infty}\left(\mathbb{R}_{t} \times\right] 0, L\left[\times \mathbb{R}_{p}^{2}\right) ; \eta\left(t, 0, p_{x}<0, p_{y}\right)=\eta\left(t, L, p_{x}>0, p_{y}\right)=0\right. \\
\left.\operatorname{supp}(\eta) \text { bounded set of } \mathbb{R}_{t} \times[0, L] \times \mathbb{R}_{p}^{2}\right\}
\end{array}
$$

Definition 4 Let $E_{x}, E_{y}, B_{z} \in L^{\infty}\left(\mathbb{R}_{t} ; W^{1, \infty}(] 0, L[)\right)$ and $g_{0}, g_{L} \in L_{\text {loc }}^{1}\left(\mathbb{R}_{t} \times\right.$ $\left.\Sigma^{-}\right)$be $T$ periodic functions. The function $f \in L_{\text {loc }}^{1}\left(\mathbb{R}_{t} \times\right] 0, L\left[\times \mathbb{R}_{p}^{2}\right)$ which is the mild periodic solution of problem (98), (102), (103) is given by (109):

$$
\begin{aligned}
<f, \varphi>= & \int_{0}^{T} d t \int_{p_{x}>0} \int_{p_{y}} d p \int_{t}^{\tau_{o}} V_{x}(p) \cdot g_{0}\left(t, p_{x}, p_{y}\right) \\
& \cdot \quad \varphi\left(s, X\left(s ; 0, p_{x}, p_{y}, t\right), P_{x}\left(s ; 0, p_{x}, p_{y}, t\right), P_{y}\left(s ; 0, p_{x}, p_{y}, t\right) d s\right. \\
& -\quad \int_{0}^{T} d t \int_{p_{x}<0} \int_{p_{y}} d p \int_{t}^{\tau_{o}} V_{x}(p) \cdot g_{L}\left(t, p_{x}, p_{y}\right) \\
& \cdot \varphi\left(s, X\left(s ; L, p_{x}, p_{y}, t\right), P_{x}\left(s ; L, p_{x}, p_{y}, t\right), P_{y}\left(s ; L, p_{x}, p_{y}, t\right) d s,\right.
\end{aligned}
$$


where $\left(X(s), P_{x}(s), P_{y}(s)\right)$ is the solution of the system:

$$
\left\{\begin{array}{lll}
\frac{d X}{d s} & =V_{x}\left(P\left(s ; x, p_{x}, p_{y}, t\right)\right), & s \in\left[\tau_{i}, \tau_{o}\right] \\
X\left(t ; x, p_{x}, p_{y}, t\right) & =x, & \\
\frac{d P_{x}}{d s} & =q \cdot\left(E_{x}(s, X(s))+V_{y}(P(s)) \cdot B_{z}(s, X(s))\right), & s \in\left[\tau_{i}, \tau_{o}\right] \\
P_{x}\left(t ; x, p_{x}, p_{y}, t\right) & =p_{x}, & \\
\frac{d P_{y}}{d s} & =q \cdot\left(E_{y}(s, X(s))-V_{x}(P(s)) \cdot B_{z}(s, X(s))\right), & s \in\left[\tau_{i}, \tau_{o}\right] \\
P_{y}\left(t ; x, p_{x}, p_{y}, t\right) & =p_{y} . &
\end{array}\right.
$$

In the relativistic case, the analogue of Lemma 1 is given by:

Lemma 4 Assume that the electro-magnetic field and the boundary data satisfy:

$$
\begin{gathered}
\|E\|_{L^{\infty}}+c \cdot\left\|B_{z}\right\|_{L^{\infty}} \leq \frac{m \cdot\left(p_{0} / m\right)^{2}}{4 q L} \cdot\left[1+\left(\frac{p_{1}+p_{0} / 2}{m c}\right)^{2}\right]^{-1 / 2} \\
\left(E_{x}, E_{y}, B_{z}\right) \in\left(L^{\infty}\left(\mathbb{R}_{t} ; W^{1, \infty}(] 0, L[)\right)\right)^{3}, \\
\operatorname{supp}\left(g_{0}\right) \subset\left\{\left(t, x, p_{x}, p_{y}\right) ; t \in \mathbb{R}_{t}, x=0,0<p_{0} \leq p_{x}, \sqrt{p_{x}^{2}+p_{y}^{2}} \leq p_{1}\right\}, \\
\operatorname{supp}\left(g_{L}\right) \subset\left\{\left(t, x, p_{x}, p_{y}\right) ; t \in \mathbb{R}_{t}, x=L, 0>-p_{0} \geq p_{x}, \sqrt{p_{x}^{2}+p_{y}^{2}} \leq p_{1}\right\} .
\end{gathered}
$$

Then, the life-time in $] 0, L\left[\right.$ of particles starting from the support of $g_{0}$ and $g_{L}$ is finite:

$$
\begin{aligned}
\tau_{o}\left(x, p_{x}, p_{y}, t\right)-\tau_{i}\left(x, p_{x}, p_{y}, t\right) & \leq 2 \cdot \frac{L \cdot m}{p_{0}} \sqrt{1+\left(\frac{p_{1}+p_{0} / 2}{m c}\right)^{2}}, \\
& \forall\left(t, x, p_{x}, p_{y}\right) \in \operatorname{supp}\left(g_{0}\right) \cup \operatorname{supp}\left(g_{L}\right) .
\end{aligned}
$$

$\mathrm{RR} \mathrm{n}^{\circ} 3554$ 
Corollary 2 Assuming the same hypotheses as in Lemma 4 and let $f$ be the mild solution of Definition 4. Then we have:

$$
\begin{gathered}
\operatorname{supp}(f) \subset\left\{\left(t, x, p_{x}, p_{y}\right)\left|t \in \mathbb{R}_{t}, x \in[0, L], \frac{p_{0}}{2} \leq\right| p_{x} \mid, \sqrt{p_{x}^{2}+p_{y}^{2}} \leq p_{1}+\frac{p_{0}}{2}\right\} \\
\|\rho\|_{L^{\infty}} \leq \frac{\pi}{2}\left(p_{1}+p_{0} / 2\right)^{2} \cdot q \cdot\left(\left\|g_{0}\right\|_{L^{\infty}}+\left\|g_{L}\right\|_{L^{\infty}}\right),
\end{gathered}
$$

and

$$
\max \left\{\left\|j_{x}\right\|_{L^{\infty}},\left\|j_{y}\right\|_{L^{\infty}}\right\} \leq c \cdot \frac{\pi}{2}\left(p_{1}+p_{0} / 2\right)^{2} \cdot q \cdot\left(\left\|g_{0}\right\|_{L^{\infty}}+\left\|g_{L}\right\|_{L^{\infty}}\right)
$$

where $\rho(t, x)=q \int_{\mathbb{R}_{p}^{2}} f\left(t, x, p_{x}, p_{y}\right) d p$ and $j_{x, y}(t, x)=q \int_{\mathbb{R}_{p}^{2}} V_{x, y}(p) f\left(t, x, p_{x}, p_{y}\right) d p$.

Like in the classical case, we first show the existence of weak periodic solution for the regularized problem by using the Schauder fixed point theorem. Next we pass to the limit when the regularization parameter vanishes. We have the following Theorem:

Theorem 2 Let $g_{0}, g_{L}, h_{0}, h_{L} \in L^{\infty}\left(\mathbb{R}_{t} \times \Sigma^{-}\right)$be $T$ periodic functions and $p_{0}, p_{1}, K$ constants which verify:

$$
\begin{gathered}
\operatorname{supp}\left(g_{0}\right) \subset\left\{\left(t, x, p_{x}, p_{y}\right) ; t \in \mathbb{R}_{t}, x=0,0<p_{0} \leq p_{x}, \sqrt{p_{x}^{2}+p_{y}^{2}} \leq p_{1}\right\}, \\
\operatorname{supp}\left(g_{L}\right) \subset\left\{\left(t, x, p_{x}, p_{y}\right) ; t \in \mathbb{R}_{t}, x=L, 0>-p_{0} \geq p_{x}, \sqrt{p_{x}^{2}+p_{y}^{2}} \leq p_{1}\right\}, \\
2 \cdot K \leq \frac{m \cdot\left(p_{0} / m\right)^{2}}{4 q L} \cdot\left[1+\left(\frac{p_{1}+p_{0} / 2}{m c}\right)^{2}\right]^{-1 / 2}, \\
\frac{1}{\varepsilon} \cdot \frac{\pi}{2} \cdot q\left(\left\|g_{0}\right\|_{L^{\infty}}+\left\|g_{L}\right\|_{L^{\infty}}\right) \cdot\left(p_{1}+p_{0} / 2\right)^{2}(c T+L)<\frac{K}{\sqrt{2}},
\end{gathered}
$$




$$
\begin{aligned}
& \frac{L}{2 \varepsilon} \cdot \frac{\pi}{2} \cdot q\left(\left\|g_{0}\right\|_{L^{\infty}}+\left\|g_{L}\right\|_{L^{\infty}}\right) \cdot\left(p_{1}+p_{0} / 2\right)^{2}+\frac{1}{2}\left(\left\|h_{0}\right\|_{L^{\infty}}+\left\|h_{L}\right\|_{L^{\infty}}\right) \leq \frac{K}{\sqrt{2}} \\
& \int_{0}^{T} d t \int_{p_{x}>0} \int_{p_{y}} V_{x}(p) g_{0}\left(t, p_{x}, p_{y}\right) d p+\int_{0}^{T} d t \int_{p_{x}<0} \int_{p_{y}} V_{x}(p) g_{L}\left(t, p_{x}, p_{y}\right) d p=0 .
\end{aligned}
$$

Then the relativistic $1 D$ Vlasov-Maxwell system has at least one weak periodic solution.

\section{References}

[1] C. Greengard and P.A. Raviart, A boundary value problem for the stationary Vlasov-Poisson system, Comm. Pure and Appl. Math. XLIII(1990), pp. $473-507$.

[2] C. Bardos, Problèmes aux limites pour les équations aux dérivées partielles du premier ordre, Ann. Sci. Ecole norm. sup., 4:185:233, (1969).

[3] R. J. Diperna et P. L. Lions, Ordinary differential equations, transport theory and Sobolev spaces, Invent. Math., 98:511:547, (1989).

[4] F. Poupaud, Boundary value problems for the stationary Vlasov-Maxwell system, Forum Math., 4(1992), pp. 499-527 and C.R.A.S., t 311, Série I, pp 307-312 (1990).

[5] Y. Guo, Regularity for the Vlasov equation in a half space, Indiana Univ. Math. J. 43(1994), pp 255-320.

[6] Y. Guo, Global weak solutions of the Vlasov-Maxwell system with boundary conditions, Comm. Math. Phys. 154(1993), pp. 245-263.

[7] B. Bodin, Modélisation et simulation numérique du régime de ChildLangmuir, Thèse de l'Ecole Polytechnique, Palaiseau (1995).

[8] K. Pfaffelmoser, Global classical solutions of the Vlasov-Poisson system in 3 dimensions for general initial data, J. Diff. Eq. 95(1992), pp. 281-303.

$\mathrm{RR} \mathrm{n}^{\circ} 3554$ 
[9] P.L. Lions and B. Perthame Propagation of moments and regularity for the 3-dimensional Vlasov-Poisson system, Invent. Math. 105(1991), pp. 415-430.

[10] N. Ben.Abdallah Weak solutions of the initial-boundary value problem for the Vlasov-Poisson system, Preprint.

[11] M. Bostan and F. Poupaud, Periodic solutions of the Vlasov-Poisson system with boundary conditions, C.R.Acad.Sci.Paris, t.325, Série I, p.13331336, 1997.

[12] M. Bostan and F. Poupaud, Periodic solutions of the Vlasov-Poisson system with boundary conditions, report INRIA-CERMICS, $N^{\circ} 3518$, (Octobre 1998).

[13] M. Bostan and F. Poupaud, Controlability methods for the calculation of periodic solutions of the Vlasov-Maxwell system with boundary conditions, report INRIA-CERMICS, $N^{\circ} 3534$, (Octobre 1998).

[14] R. J. Diperna et P. L. Lions, Global weak solutions of Vlasov-Maxwell system, Comm. Pure Appl. Math. XVII(1989), pp. 729-757.

[15] M. Bezard, Boundary value problems for the Vlasov-Maxwell system, Semin. Equ. Deriv. Partielles, Ec. Polytech., Cent. Math., Palaiseau Semi 1992-1993, Exp. No.4, pp. 17 (1993).

[16] A. Arsenev, Global existence of a weak solution of Vlasov's system of equations, USSR Comp. Math. and Math. Phys. 15, 1975, pp. 131-143.

[17] P. Degond, Regularité de la solution des équations cinétiques en physiques de plasmas, [J] Semin., Equations Deriv. Partielles 1985-86, Expose No.18, 11 p. (1986).

[18] P. Degond, Local existence of solutions of the Vlasov-Maxwell equations and convergence to the Vlasov-Poisson equations for infinite light velocity, [J] Math. Methods Appl. Sci. 8, 533-558 (1986). 
[19] P. Degond, Global existence of smooth solutions for the Vlasov-FokkerPlanck equation in 1 and 2 space dimensions, [J] Ann. Sci. Ec. Norm. Super., IV. Ser. 19, 519-542 (1986).

[20] K. Asano and S. Ukai, On the Vlasov-Poisson limit of the Vlasov-Maxwell equation, [CA] Pattern and waves. Qualitative analysis of nonlinear differential equations, Stud. Math. Appl. 18, 369-383 (1986).

$\mathrm{RR} \mathrm{n}^{\circ} 3554$ 


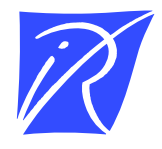

Unité de recherche INRIA Sophia Antipolis 2004, route des Lucioles - B.P. 93 - 06902 Sophia Antipolis Cedex (France)

Unité de recherche INRIA Lorraine : Technopôle de Nancy-Brabois - Campus scientifique 615, rue du Jardin Botanique - B.P. 101 - 54602 Villers lès Nancy Cedex (France)

Unité de recherche INRIA Rennes : IRISA, Campus universitaire de Beaulieu - 35042 Rennes Cedex (France)

Unité de recherche INRIA Rhône-Alpes : 655, avenue de l'Europe - 38330 Montbonnot St Martin (France)

Unité de recherche INRIA Rocquencourt : Domaine de Voluceau - Rocquencourt - B.P. 105 - 78153 Le Chesnay Cedex (France)

Éditeur

INRIA - Domaine de Voluceau - Rocquencourt, B.P. 105 - 78153 Le Chesnay Cedex (France)

http://www.inria.fr

ISSN 0249-6399 